\title{
Spread and Control of Multi-drug Resistance Tuberculosis and Drug-sensitive Tuberculosis in Ethiopia: A Mathematical Model Analysis
}

\author{
Shimelis Bekele Zerefe ${ }^{1}$, Temesgen Tibebu Mekonnen ${ }^{2, *}$ \\ ${ }^{1}$ Department of Mathematics, Addis Ababa Science and Technology University, Addis Ababa, Ethiopia \\ ${ }^{2}$ Department of Mathematics, Debre Berhan University, Debre Berhan, Ethiopia \\ Email address: \\ shimelis.bekele@aastu.edu.et (S. B. Zerefe), temesgentibebumekonnen@yahoo.com (T. T. Mekonnen) \\ ${ }^{*}$ Corresponding author
}

To cite this article:

Shimelis Bekele Zerefe, Temesgen Tibebu Mekonnen. Spread and Control of Multi-drug Resistance Tuberculosis and Drug-sensitive Tuberculosis in Ethiopia: A Mathematical Model Analysis. American Journal of Applied Mathematics. Vol. 8, No. 3, 2020 , pp. $123-144$. doi: 10.11648/j.ajam.20200803.15

Received: April 22, 2020; Accepted: May 15, 2020; Published: May 29, 2020

\begin{abstract}
In this work we considered nonlinear dynamical system to study the dynamics of two-strain Tuberculosis epidemic in Ethiopia. We proved that the solution of the considered dynamical system is positive and bounded. We found that the considered dynamical system has disease free and endemic equilibrium points. We proved that the local and global stability of disease free equilibrium point and endemic equilibrium point. We found the effective reproduction number of the dynamical system. Also, the effective reproduction number of the dynamical system which experience drug sensitive strain and the effective reproduction number of the dynamical system which experience multi drug resistance strain. Using real data collected from different health sectors from Ethiopia we found that the numerical value of the effective reproduction number of the drug sensitive tuberculosis is 1.03 and the effective reproduction number of the drug resistance tuberculosis is 4.78 and the effective reproduction number of the dynamical system $\max \{1.03,4.78\}=4.78$. So that MDR strain is spreads strongly than DS strain. Numerical simulation is also done to illustrate the influence of different parameters on the effective reproduction number. Using sensitive analysis we identify the most influential parameter to change the behavior of the solution of the considered dynamical system is the number of effective contacts of susceptible or vaccinated individuals make with an infectious individual.
\end{abstract}

Keywords: Drug-sensitive Tuberculosis, Drug Resistance Tuberculosis, Effective Reproduction Number, Sensitivity Analysis, Numerical Analysis

\section{Introduction}

Tuberculosis is among the most ancient diseases. German Microbiologist Robert Koch discovered the causative organism Mycobacterium tuberculosis on $24^{\text {th }}$ March 1882. World Health Organization (WHO) declared tuberculosis as global epidemic in 1993 [1, 2]. The lifetime risk of TB reactivation for a person with documented Latent Tuberculosis Infection (LTBI) is estimated to be $5 \%-10 \%$, with the majority developing TB disease within the first five years after initial infection the risk of developing TB disease following infection depends on several factors $[1,3]$. There is a huge TB-latent human; this increased its average probability of re-activation due to the emergence and growth
HIV and TB drug-resistant strains [1]. One of the biggest health challenges facing the world is tied in to the dramatic increases in the levels of drug resistance TB, particular in hospital settings [1, 4]. In 2016, the World Health Organization (WHO) reports roughly 9.4 million new cases (incidence) per year, an active-TB prevalence of 14 million, and 1.6 to 1.9 million deaths per year, a number that includes 400,000 deaths coming from HIV positive individuals each year. Most active-TB cases are concentrated in South East Asia, African and Western Pacific regions [1,5]. In Ethiopia there were in average of $177 \mathrm{~TB}$ cases per $100,000 \mathrm{~TB}$ in $2016[5,6,7,8]$.

Currently, WHO recommends that, the countries use three major categories of health interventions for TB prevention: 
treatment of LTBI; prevention of transmission of Mycobacterium tuberculosis through infection control; and vaccination of children with the Bacille Calmette-Guérin (BCG) vaccine. In 2016, 154 countries reported providing BCG vaccination as a standard part of these Programmes, of which 111 reported coverage above 90\% [5]. Results of field trials of the BCG vaccine have differed widely, some indicating protection rates as high as $70 \%$ to $80 \%$, others indicating the vaccine was completely ineffective in preventing TB [4].

Drug Susceptibility Sesting (DST) is very important to provide information about which drugs a person is resistant to. Treatment of tuberculosis disease is not simple and Drug Susceptible Tuberculosis (DS-TB) requires a multiple drug regimen taken for at least six months. But the treatment will only be successful if the drugs are taken exactly as required for the entire length of time [5]. The currently recommended treatment for cases of drug-susceptible TB must be faithfully carried out over 6-9 months regimen of four first-line drugs: isoniazid, rifampicin, ethambutol and pyrazinamide and is a source of concern due the fact that a number of TB-active individuals do not complete treatment giving rise to the emergence of drug resistance TB strains $[1,5]$. Treatment for rifampicin-resistant TB (RR-TB) and multidrugresistant tuberculosis (MDR-TB) is longer, and requires more expensive and more toxic drugs [5].

Multi-drug-resistant (MDR) tuberculosis is a form of tuberculosis caused by bacteria that do not respond to, at least, isoniazid and rifampicin, which are the two most powerful, standard anti-tuberculosis drugs [3, 9, 10]. According to the world health organization (WHO) global TB report in 2017, it is estimated that there will be 490,000 new cases of Multi-Drug Resistant Tuberculosis (MDR-TB) in 2016, in addition, 110,000 new patients who resistant to rifampicin meet the treatment conditions of multi drug resistance tuberculosis [5, 11]. A combination of poor compliance and poor medical supervision or when the antiTB drugs are mismanaged (incomplete course of treatment) or misused (wrong dose or time length to complete the drugs) can result multi-drug resistance. However some acquire MDR-TB by being infected with a multi-drug resistant strain. MDR-TB is transmitted in the same way as the normal drug sensitive strain [3, 11, 12]. Drug-resistant TB has a higher mortality rate, among them, multi-drug resistant tuberculosis (MDR-TB) is more prominent, and has become another new serious problem [11]. Multi-drugresistant tuberculosis (MDR-TB) treatment regimens are significantly longer, cause serious side effects and are very expensive. The latest data reported to WHO show a treatment success rate for multi-drug resistant tuberculosis (MDR-TB) of 54\%, globally, reflecting high rates of loss to follow-up, unevaluated treatment outcomes and treatment failure [5, 12] and TB treatment outcomes in Ethiopia have been assessed only in small and fragmented observational studies [13].

In this work we present a non-linear dynamical system to study the spread and control of the dynamics of tuberculosis in Ethiopia which describes the infectious disease of two strains tuberculosis. The interventions: vaccination, screening and treatments are incorporated in our model. Based on the Ethiopia context we introduced the model assumptions, construct flow chart of the model and develop the corresponding dynamical system. We investigated disease free equilibrium point, endemic equilibrium points and effective reproduction number. We showed that their local and global stability. Numerical simulations of the results are done by real data collected from different health sectors in Ethiopia. Finally we suggest our recommendation based on the finding of our work which is MDR strain is spreads strongly than DS strain.

\section{The Initial Model}

In the work [14], the total population $N(t)$ was divided in to eight classes: Susceptible S (t),; Vaccinated V (t), early stage with high risk of developing active tuberculosis $H_{r}(t)$ and Later (Long) stage with low risk of developing active disease $L_{r}(t)$, individuals who screened and treating at early latent stage tuberculosis $T(t)$, Infectious individuals with tuberculosis $I(t)$, treating infectious $I_{T}(t)$ and Recovered individuals $R(t)$, recruitment of the population $\Lambda$ with the proportions $\psi$ of which are vaccinated to protect them against tuberculosis infection and the remaining proportion are susceptible, force of infection rate $\lambda=c \omega\left(\frac{I}{N}\right)$ and $\omega$ is the probability that an individual is infected by one infectious individual, and $c$ is the contact rate. The proportion $p$ of class $H_{r}$ have got a chance of screened. The proportion $\varepsilon$ and $(1-\varepsilon)$ of individuals of the early latent/exposed individuals for tuberculosis who do not get chance for screened will go to $L_{r}$ and $I$ respectively at the rate $\alpha$. Individual leaves class $L_{r}$ at the rate $\gamma$ in which, the proportion $\delta$ goes to class $I$; the proportion $(1-\delta)$ recovers naturally and enter to recovered class R. The proportion $q$ of individuals in class $I$ goes for treatment in $I_{T}$ and the remaining proportion $(1-q)$ enters to class $R$ at the rate $\rho$. Individuals leave the screened class $T$ and treating class $I_{T}$ at the rates $\phi$, and $\varphi$ respectively, and go to recovered class. $\kappa$ is the reduction in susceptibility of recovered individual. Natural death at the rate $\mu$ while infectious individuals in $I$ are die due to tuberculosis diseases at the rate $d$.

The corresponding dynamical system of the figure 1 is

$$
\begin{aligned}
\frac{d V}{d t} & =\psi \Lambda-(\sigma \lambda+\theta+\mu) V \\
\frac{d s}{d t} & =(1-\psi) \Lambda+\theta V-(\lambda+\mu) S \\
\frac{d H_{r}}{d t} & =\lambda(S+\sigma V+\kappa R)-(\alpha+\mu) H_{r} \\
\frac{d L_{r}}{d t} & =\alpha \varepsilon(1-p) H_{r}-(\gamma+\mu) L_{r} \\
\frac{d T}{d t} & =\alpha p H_{r}-(\phi+\mu) T \\
\frac{d I}{d t}=\delta \gamma L_{r}+\alpha & (1-\varepsilon)(1-p) H_{r}-(\rho+\mu+d) I \\
\frac{d I_{T}}{d t} & =q \rho I-(\varphi+\mu) I_{T}
\end{aligned}
$$




$$
\frac{d R}{d t}=\phi T+(1-q) \rho I+(1-\delta) \gamma L_{r}-(\kappa \lambda+\mu) R(8)
$$

With the total population at a given time $\mathrm{t}$ is $N(t)=$ $S(t)+V(t)+H_{r}(t)+L_{r}(t)+I(t)+T(t)+R(t)$.
We extend the model in [26] by introducing additional assumptions as follows:

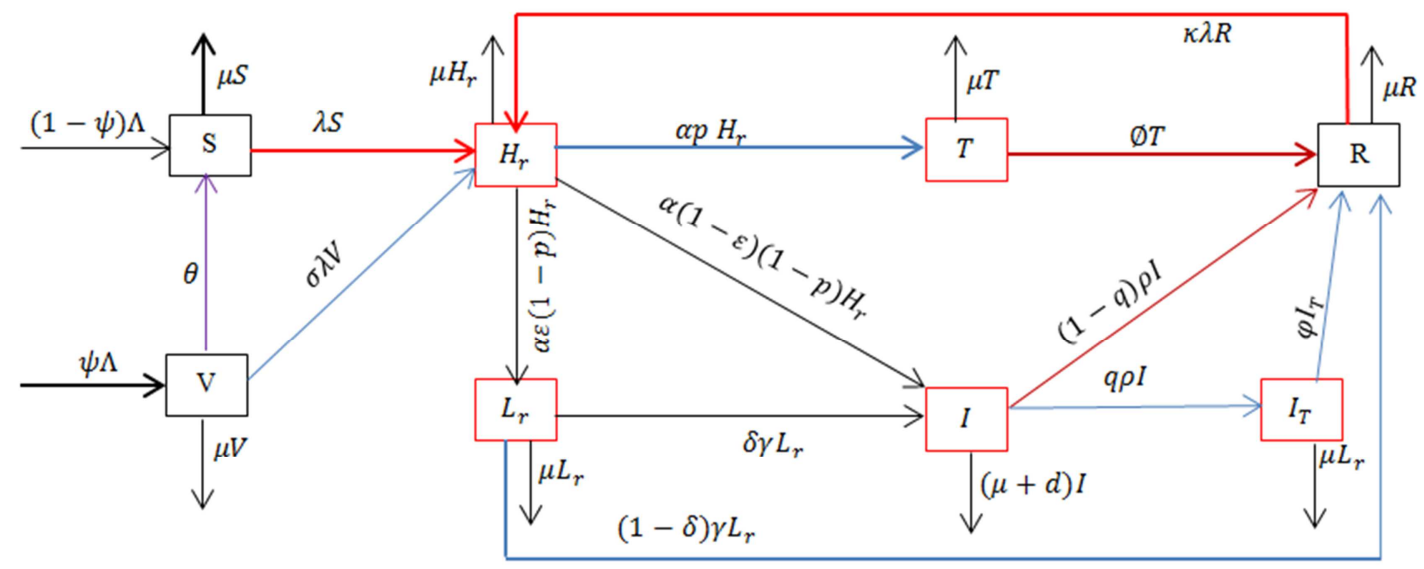

Figure 1. Flow chart of the dynamical system of tuberculosis.

\section{The extended Model}

\subsection{Model Assumptions}

In this study, we introduce a deterministic TB model by disaggregating the mycobacterium tuberculosis in to two strains (DS-TB, MDR-TB). The total population $N(t)$ is divided in to ten disjoint classes depending on the epidemiological status of individuals such as: Susceptible $S(t)$, who have never exposed to any strain of the Mycobacterium tuberculosis, Vaccinated $V(t)$, who have taken $\mathrm{BCG}$ vaccine against mycobacterium tuberculosis, an early stage infected with high risk of developing active drug sensitive tuberculosis $H_{s}(t)$ and Later (Long) stage infected with low risk of developing active drug sensitive tuberculosis $L_{S}(t)$, Infectious individuals with drug sensitive tuberculosis $I_{s}(t)$, Latently infected individuals with multi-drug resistant tuberculosis $E(t)$, Infectious individuals with multi-drug resistant tuberculosis $I_{r}(t)$, Screened Early latently infected with drug sensitive tuberculosis $T_{s}(t)$, Screened latently infected with multi-drug resistant tuberculosis $T_{r}(t)$ and Recovered individuals $R(t)$.

Assume that individuals are recruited into the population by a constant rate $\Lambda$ with the proportions $\psi$ of which are vaccinated to protect them against tuberculosis infection. Furthermore, that the vaccine has a waning effect over time (after a time $\frac{1}{\theta}$ vaccinated individuals become susceptible again) and reduces due to expiration of duration of vaccine efficacy. We assume that vaccinated individuals may infect with the rate of inefficacy of vaccine $\sigma \epsilon[0,1]$. Susceptible population increases due to the coming in of new births not vaccinated against the infection and those who were vaccinated but lose their immunity. When some susceptible individuals come into contact with infectious individuals, they get infected and progress to latently infected classes of drug susceptible and multi-drug resistant tuberculosis at a force of infection rates $\lambda_{s}$ and $\lambda_{r}$ respectively where $\lambda_{i}=c \omega_{i}\left(\frac{I_{i}}{N}\right), i=s, r$ and $\omega_{i}$ is the probability that an individual is infected by one infectious individual, and $c$ is the number of effective contacts.

The proportion $p$ of the high risk latently drug susceptible TB infected developed active TB and the remaining proportion enters to long latent with drug sensitive TB at the rate $\alpha$. The proportion $\varepsilon$ and $(1-\varepsilon)$ of individuals of the early latent/exposed individuals for drug susceptible tuberculosis who do not get chance for screened will go to $L_{s}$ and $I$ respectively at the rate $\alpha$. Thus, the proportion $p, \varepsilon(1-p)$ and $(1-\varepsilon)(1-p)$ of individuals in the class $H_{s}$ is transferred to classes $T_{s}, L_{s}$ and $I_{s}$ respectively at a rate $\alpha$. Individual leaves class $L_{s}$ at the rate $\gamma$ in which, the proportion $\eta$ goes to class $I_{s}$ and; the remaining proportion $(1-\eta)$ recovers naturally and enter to recovered class R.

Individuals in $H_{s}$ and, $L_{s}$ can also be infected by MDR TB (primary infection) if there is effective contact with individuals in $I_{r}$ class. Individuals leave $I_{s}$ class at the rates $\rho_{s}$ that the proportion $q$ of individuals in infectious classes of drug susceptible tuberculosis progress to the recovered class while the remaining $(1-q)$ proportion of individuals with active drug sensitive TB may develop MDR TB because of improper treatment.

The proportion $v$ of the latently infected multi-drug resist tuberculosis are screened for treatment and the remaining proportion developed active drug resist tuberculosis and leaves $\mathrm{E}$ class at the rate of $\delta$. Individual in $I_{r}$ class recovers at the rate $\rho_{r}$ and goes to $\mathrm{R}$ class. Individuals leave the screened classes $T_{s}$ and $T_{r}$ at the rates $\phi$, and $\varphi$ respectively, and go to recovered class, where $\phi>\varphi$.

Due to the nature of the disease, the infection will only kill individuals whose TB progresses to the infectious stage. Moreover, individuals in the recovered class are temporarily recovered. Soon they revert back to the latently infected classes $H_{s}$ and $E$, after been re-infected by either drug sensitive class and multi-drug resistant strain at the rate $\kappa \lambda_{s}$ and $\kappa \lambda_{r}$ respectively, where $\kappa$ is the reduction in 
susceptibility due to prior endogenous infection of tuberculosis. We assume that each class conforms to natural death at the rate $\mu$ while infectious individuals in $I_{s}$ and $I_{r}$ are die due to TB diseases at the rate $d_{s}$ and $d_{r}$ respectively. State variables and parameters in the dynamical system listed in the following table.

Table 1. Symbols and there description for state variables and parameters.

\begin{tabular}{|c|c|}
\hline Symbols & Description \\
\hline$V$ & Vaccinated individuals against tuberculosis disease. \\
\hline$S$ & Susceptible individuals for the disease \\
\hline$H_{s}$ & Early latently infected with drug sensitive tuberculosis \\
\hline$I_{s}$ & Infectious individuals with drug sensitive tuberculosis \\
\hline$T_{s}$ & Screened Early latently infected with drug sensitive tuberculosis \\
\hline E & Latently infected with multi-drug resistant tuberculosis \\
\hline$T_{r}$ & Screened Latently infected with multi-drug resistant tuberculosis \\
\hline $\mathrm{R}$ & Recovered Individuals \\
\hline$\Lambda$ & Recruitment of population \\
\hline$\psi$ & Proportions new born vaccinated \\
\hline$\mu$ & Natural death rate \\
\hline$\sigma$ & the rate of inefficacy of vaccine individuals \\
\hline$\theta$ & the rate of vaccine waning \\
\hline$\lambda_{i}(i=s, r)$ & Force of infection ( $s=$ DS strain, $r=$ MDR strain) \\
\hline$c$ & Number of effective contacts susceptible or vaccinated individuals makes with infectious individuals. \\
\hline$\alpha$ & The rate of progression of individuals from early latently infected with DS-TB. \\
\hline$d_{i}(i=s, r)$ & Death rate due to the disease ( $s=$ DS strain, $r=$ MDR strain) \\
\hline$p$ & Proportion of latently infected DS- TB at early stage for treatment \\
\hline$\varepsilon$ & Proportion of individuals who do not get chance for screened at $H_{S}$ and will go to $L_{S}$ class. \\
\hline$\phi$ & Rate of individuals move from $T_{s}$ to $\mathrm{R}$ \\
\hline$q$ & Proportion of infectious individuals with DS-TB who enters to recovered class. \\
\hline$\gamma$ & Progression rate from Long latently infected with DS-TB strain. \\
\hline$\delta$ & Progression rate from latency MDR-TB. \\
\hline$\varphi$ & Rate of individuals move from $T_{r}$ to $\mathrm{R}$ \\
\hline$\eta$ & The portion of $L_{S}$ enter in to $I_{S}$ \\
\hline$v$ & The portion of $E$ enter in to $I_{r}$ \\
\hline$\rho_{i}(i=s, r)$ & The recovery rate infectious individuals ( $s=\mathrm{DS}$ strain, $r=$ MDR strain). \\
\hline$\kappa$ & Acquired immunity due to previous treatment. \\
\hline
\end{tabular}

Based on the above assumptions we do have the following flow chart:

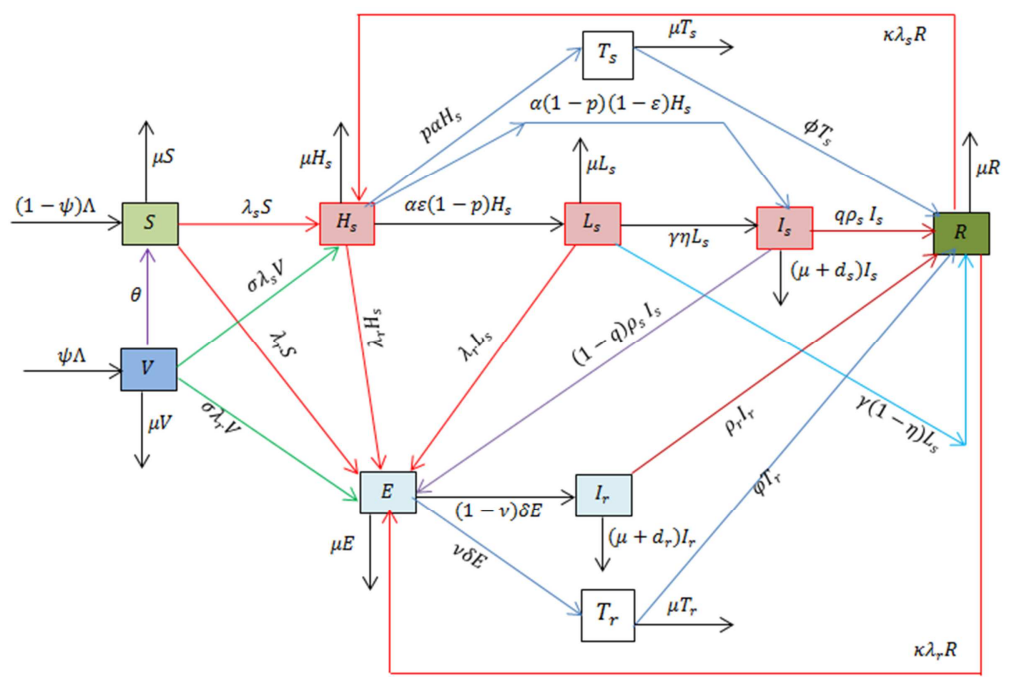

Figure 2. Flow diagram of the dynamical system (9)-(18).

Based on the assumptions and the above flow chart we develop the following nonlinear dynamical system.

$$
\begin{gathered}
\frac{d V}{d t}=\psi \Lambda-\left(\sigma\left(\lambda_{s}+\lambda_{r}\right)+\theta+\mu\right) V \\
\frac{d s}{d t}=(1-\psi) \Lambda+\theta V-\left(\lambda_{s}+\lambda_{r}+\mu\right) S
\end{gathered}
$$




$$
\begin{gathered}
\frac{d H_{s}}{d t}=\lambda_{s}(S+\sigma V+\kappa R)-\left(\alpha+\lambda_{r}+\mu\right) H_{s} \\
\frac{d L_{s}}{d t}=\alpha \varepsilon(1-p) H_{s}-\left(\lambda_{r}+\gamma+\mu\right) L_{s} \\
\frac{d I_{s}}{d t}=\gamma \eta L_{s}+\alpha(1-\varepsilon)(1-p) H_{s}-\left(\rho_{s}+\mu+d_{s}\right) I_{s} \\
\frac{d T_{s}}{d t}=\alpha p H_{s}-(\phi+\mu) T_{s} \\
\frac{d E}{d t}=\lambda_{r}\left(S+H_{s}+L_{s}+\sigma V+\kappa R\right)+(1-q) \rho_{s} I_{s}-(\delta+\mu) E \\
\frac{d I_{r}}{d t}=(1-v) \delta E-\left(\rho_{r}+\mu+d_{r}\right) I_{r} \\
\frac{d T_{r}}{d t}=v \delta E-(\varphi+\mu) T_{r} \\
\frac{d R}{d t}=q \rho_{s} I_{s}+\rho_{r} I_{r}+\gamma(1-\eta) L_{s}+\phi T_{s}+\varphi T_{r}-\left(\kappa\left(\lambda_{s}+\lambda_{r}\right)+\mu\right) R
\end{gathered}
$$

With the total population at a given time $t$ :

$$
N(t)=V(t)+S(t)+H_{S}(t)+L_{S}(t)+I_{S}(t)+T_{S}(t)+E(t)+I_{r}(t)+T_{r}(t)+R(t)
$$

\subsection{Positivity of Solutions of the Dynamical System (9) - (18)}

Theorem-1:

Let the initial data for the model (9)-(18) be $V(0)>0, S(0)>0, H_{S}(0)>0, L_{S}(0)>0, I_{S}(0)>0, T_{S}(0)>0, E(0)>$ $0, I_{r}(0)>0, T_{r}(0)>0$ and $R(0)>0$. Then, the solutions $V(t), S(t), H_{s}(t), L_{s}(t), I_{s}(t), T_{s}(t), E(t), I_{r}(t), T_{r}(t)$ and $R(t)$ of the model (9)-(18) will be remain positive for all time $t>0$.

Proof:

Let $\quad \bar{t}=\sup \left\{t>0: V(0)>0, S(0)>0, H_{S}(0)>0, L_{S}(0)>0, I_{S}(0)>0, T_{S}(0)>0, E(0)>0, I_{r}(0)>0, T_{r}(0)>\right.$ 0 and $R(0)>0\} \in[0, t]$.

From the dynamical system (9) - (18) of the system, we solve for the state variable:

$$
V(t)=V(0) Q_{1}+Q_{1} \int_{0}^{\bar{t}} \psi \Lambda \exp \left\{\mu t+\theta t+\int_{0}^{w}\left(\sigma\left(\lambda_{s}+\lambda_{r}\right)(\tau)\right) d \tau\right\} d t>0
$$

Where $Q_{1}=\exp -\left\{\mu t+\theta t+\int_{0}^{\bar{t}} \sigma\left(\lambda_{s}+\lambda_{r}\right)(\tau) d \tau\right\}>0$

$$
\left.S(t)=S(0) Q_{2}+Q_{2} \int_{0}^{\bar{t}}((1-\psi) \Lambda+\theta V(t)) \int_{0}^{\bar{t}}((1-\psi) \Lambda+\theta V(t)) \exp \left[\mu t+\int_{0}^{\bar{w}}\left(\lambda_{s}+\lambda_{r}\right)(\tau)\right) d \tau\right] d t>0,
$$

Where $\left.Q_{2}=\exp -\left[\mu t+\int_{0}^{\bar{t}}\left(\lambda_{s}+\lambda_{r}\right)(\tau)\right) d \tau\right]>0$

$$
H_{S}(t)=H_{S}(0) Q_{3}+Q_{3} \int_{0}^{\bar{t}}\left[\left(\lambda_{S} S(t)+\sigma \lambda_{s} V(t)+\kappa \lambda_{s} R(t)\right)\left(\alpha \bar{t}+\mu \bar{t}+\int_{0}^{\bar{t}} \lambda_{r}(\tau) d \tau\right)\right] d t>0
$$

Where $Q_{3}=\exp -\left[\alpha \bar{t}+\mu \bar{t}+\int_{0}^{\bar{t}} \lambda_{r}(\tau) d \tau\right]>0$

$$
L_{s}(t)=L_{s}(0) Q_{4}+Q_{4} \int_{0}^{\bar{t}} \alpha \varepsilon(1-p) H_{s} \exp \left[\gamma \bar{t}+\mu \bar{t}+\int_{0}^{\bar{t}} \lambda_{r}(\tau) d \tau\right] d t>0
$$

Where $Q_{4}=\exp -\left[\gamma \bar{t}+\mu \bar{t}+\int_{0}^{\bar{t}} \lambda_{r}(\tau) d \tau\right]>0$

$$
I_{s}(t)=I_{s}(0) Q_{5}+Q_{5} \int_{0}^{\bar{t}}\left[\gamma \eta L_{s}+\alpha(1-\varepsilon)(1-p) H_{s}\right] \exp \left[\rho_{s} \bar{t}+d_{1} \bar{t}+\mu \bar{t}\right] d t>0
$$

Where $Q_{5}=\exp -\left[\rho_{s} \bar{t}+d_{1} \bar{t}+\mu \bar{t}\right]>0$

$$
T_{S}(t)=T_{s}(0) Q_{6}+Q_{6} \int_{0}^{\bar{t}} \alpha p H_{s} \exp [\phi \bar{t}+\mu \bar{t}] d t>0, \text { Where } Q_{6}=\exp -[\phi \bar{t}+\mu \bar{t}]>0
$$




$$
E(\bar{t})=E(0) Q_{7}+Q_{7} \int_{0}^{\bar{t}}\left[\lambda_{r}(t)\left(S+H_{s}+L_{S}+\sigma V+\kappa R\right)+(1-q) \rho_{s} I_{s}\right] \exp [\varphi \bar{t}+\mu \bar{t}] d t>0,
$$

where $Q_{7}=\exp -[\delta \bar{t}+\mu \bar{t}]>0$

$$
I_{r}(t)=I_{r}(0) Q_{8}+Q_{8} \int_{0}^{\bar{t}}[(1-v) \delta E] \exp \left[\rho_{r} \bar{t}+\mu \bar{t}+d_{2} \bar{t}\right] d t>0
$$

Where $Q_{8}=\exp -\left[\rho_{r} \bar{t}+\mu \bar{t}+d_{2} \bar{t}\right]>0$

$$
\begin{gathered}
T_{r}(t)=T_{r}(0) Q_{9}+Q_{9} \int_{0}^{\bar{t}}[v \delta E] \exp (\varphi \bar{t}+\mu \bar{t})>0, \text { Where } Q_{9}=\exp -(\varphi \bar{t}+\mu \bar{t})>0 \\
R(t)=R(0) Q_{10}+Q_{10} \int_{0}^{\bar{t}}\left[q \rho_{s} I_{s}+\rho_{r} I_{r}+\gamma(1-\eta) L_{s}+\phi T_{s}+\varphi T_{r}\right] \exp \left[\mu t+\kappa \int_{0}^{\bar{t}}\left(\lambda_{s}+\lambda_{r}\right)(\tau) d \tau\right] d t>0,
\end{gathered}
$$

Where $Q_{10}=\exp -\left[\mu t+\kappa \int_{0}^{\bar{t}}\left(\lambda_{s}+\lambda_{r}\right)(\tau) d \tau\right]>0$

Therefore all of the state variables of the dynamical system (9)-(18) are positive for all $t>0$, given any positive initial conditions.

\subsection{Positively Invariant}

Theorem-2:

The dynamical system (9) - (18) is positively invariant in the closed invariant set $\Omega=\left\{\left(V, S, H_{S}, L_{S}, I_{S}, T_{S}, E, I_{r}, T_{r}, R\right) \in\right.$ $\left.\mathbb{R}_{+}^{10}: N \leq \frac{\Lambda}{\mu}\right\}$.

Proof

Consider the biologically feasible region, $\Omega$ and observe that the rate of change of the total population obtained by adding all the equations (9)-(18) of the model. $\frac{d N}{d t}=\Lambda-\mu N-\left(d_{S} I_{s}+I_{r} d_{r}\right)$. For $N>\frac{\Lambda}{\mu}$ we do have $\frac{d N}{d t}<0$. Furthermore using a standard comparison theorem $\frac{d N}{d t} \leq \Lambda-\mu \mathrm{N}$ it follows that $\int \frac{d N}{\Lambda-\mu N} \leq \int d t \Leftrightarrow-\frac{1}{\mu} \ln (\Lambda-\mu N) \leq t+c$ where $\mathrm{c}$ is a constant $\Leftrightarrow$ $-\mu N \geq A e^{-\mu t}$, where $A=e^{-c \mu}$ is a constant. And then applying the initial condition $N(0)$ we do have $\Lambda-\mu \mathrm{N}(0) \geq \mathrm{A}$ that is $\mathrm{N}(0) \leq \frac{\Lambda}{\mu}$. Then from the inequality $\Lambda-\mu \mathrm{N} \geq \mathrm{A} e^{-\mu t}$ and taking $\mathrm{A}=\Lambda-\mu \mathrm{N}(0)$, we get $N(t) \leq \frac{\Lambda}{\mu}-\frac{\mathrm{A}}{\mu} e^{-\mu t} \leq \frac{\Lambda}{\mu}-$ $\left(\frac{\Lambda}{\mu}-\mathrm{N}(0)\right) e^{-\mu t}$. For time $t>0$ we do have $\lim _{t \rightarrow \infty} N(t) \leq N(0) e^{-\mu t}-\frac{\Lambda}{\mu} e^{-\mu t}+\frac{\Lambda}{\mu} \leq \frac{\Lambda}{\mu}$ (Since, $\left.N(0) \leq \frac{\Lambda}{\mu}\right)$. Hence if $N(0) \leq \frac{\Lambda}{\mu}$, as $t \rightarrow \infty$ the population size $N(t) \rightarrow \frac{\Lambda}{\mu}$ which implies that $0 \leq N(t) \leq \frac{\Lambda}{\mu}$. Therefore all feasible solutions of the dynamical system with initial conditions in $\Omega=\left\{\left(V, S, H_{s}, L_{s}, T_{S}, I_{S}, E, I_{r}, T_{r}, R\right) \in \mathcal{R}_{+}^{10}: N \leq \frac{\Lambda}{\mu}\right\}$ do remain in $\Omega$ for all $t>0$. That is the set $\Omega$ is positively invariant.

\subsection{Existence of Disease Free Equilibrium Point $E^{0}=\left(V^{0}, S^{0}, H_{s}{ }^{0}, L_{s}{ }^{0}, I_{s}{ }^{0}, T_{s}{ }^{0}, E^{0}, I_{r}{ }^{0}, T_{r}{ }^{0}, R^{0}\right)$}

The disease free equilibrium point of the dynamical system (9)-(18) is obtained by setting $\frac{d V}{d t}=\frac{d S}{d t}=\frac{d H_{S}}{d t}=\frac{d L_{s}}{d t}=\frac{d T_{S}}{d t}=$ $\frac{d I_{S}}{d t}=\frac{d E}{d t}=\frac{d I_{r}}{d t}=\frac{d T_{r}}{d t}=\frac{d R}{d t}=0$ and since there is no disease we do have $I_{S}=I_{r}=0$. Then the disease free equilibrium of the dynamical system (9)-(18) is,

$$
E^{0}=\left(V^{0}, S^{0}, H_{s}{ }^{0}, L_{s}{ }^{0}, I_{s}{ }^{0}, T_{s}{ }^{0}, E^{0}, I_{r}{ }^{0}, T_{r}{ }^{0}, R^{0}\right) .=\left(\frac{\psi \Lambda}{\mu+\theta}, \frac{(\theta+(1-\psi) \mu) \Lambda}{\mu(\mu+\theta)}, 0,0,0,0,0,0,0,0\right) .
$$

\subsection{Effective Reproduction Number $\boldsymbol{R}_{\text {eff }}$}

We compute the effective reproduction number $R_{\text {eff }}$ using next generation operator method. In the dynamical system (9) (18) the rate of appearance of new infections $\mathcal{F}$ and the transfer rate of individuals $\mathcal{V}$ at the disease free equilibrium point $\left(V^{0}, S^{0}, H_{s}{ }^{0}, L_{s}{ }^{0}, I_{s}{ }^{0}, T_{s}{ }^{0}, E^{0}, I_{r}{ }^{0}, T_{r}{ }^{0}, R^{0}\right)=\left(\frac{\psi \Lambda}{\mu+\theta}, \frac{(\theta+(1-\psi) \mu) \Lambda}{\mu(\mu+\theta)}, 0,0,0,0,0,0,0,0\right)$ are given as:

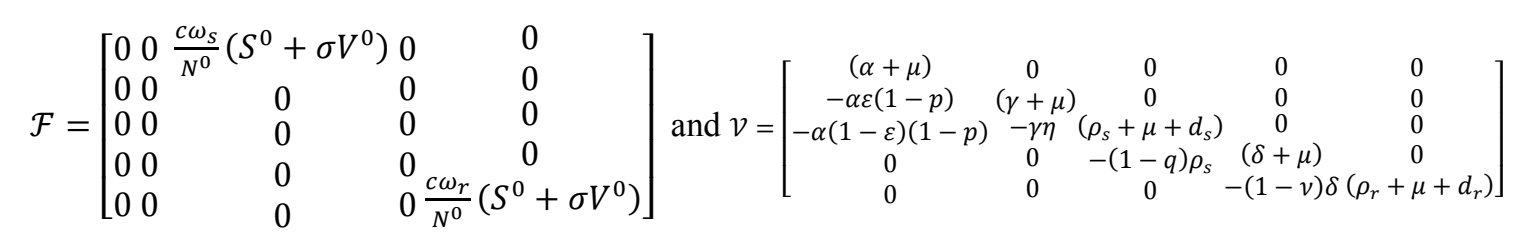


And the inverse of the matrix $v$ is:

$$
v^{-1}=\left[\begin{array}{lllll}
v_{11} & v_{21} & v_{31} & v_{41} & v_{51} \\
v_{12} & v_{22} & v_{32} & v_{42} & v_{52} \\
v_{13} & v_{23} & v_{33} & v_{43} & v_{53} \\
v_{14} & v_{24} & v_{34} & v_{44} & v_{54} \\
v_{15} & v_{25} & v_{35} & v_{45} & v_{55}
\end{array}\right]
$$

Where

$$
\begin{gathered}
v_{21}=v_{24}=v_{31}=v_{32}=v_{41}=v_{42}=v_{43}=v_{51}=v_{52}=v_{53}=v_{54}=0, v_{11}=\frac{1}{(\alpha+\mu)}, v_{12}=\frac{\alpha \varepsilon(1-p)}{(\alpha+\mu)(\gamma+\mu)}, \\
v_{13}=\frac{(\alpha \varepsilon(1-p) \gamma \eta+\alpha(\gamma+\mu)(1-\varepsilon)(1-p))}{(\alpha+\mu)(\gamma+\mu)\left(\rho_{s}+\mu+d_{s}\right)}, v_{22}=\frac{1}{(\gamma+\mu)}, v_{23}=\frac{\gamma \eta}{(\gamma+\mu)\left(\rho_{s}+\mu+d_{s}\right)}, v_{14}=\frac{(1-q) \rho_{s}[\gamma \eta \alpha \varepsilon(1-p)+\alpha(1-\varepsilon)(1-p)(\gamma+\mu)]}{(\alpha+\mu)(\gamma+\mu)\left(\rho_{s}+\mu+d_{s}\right)(\delta+\mu)\left(\rho_{r}+\mu+d_{r}\right)}, v_{33}= \\
\frac{1}{\left(\rho_{s}+\mu+d_{s}\right)}, v_{34}=\frac{(1-q) \rho_{s}}{\left(\rho_{s}+\mu+d_{s}\right)(\delta+\mu)}, v_{44}=\frac{1}{(\delta+\mu)}, \\
v_{15}=\frac{(1-q) \rho_{s}(\alpha \varepsilon(1-p) \gamma \eta+\alpha(1-\varepsilon)(1-p)(\gamma+\mu))}{(\alpha+\mu)(\gamma+\mu)\left(\rho_{s}+\mu+d_{s}\right)\left(\rho_{r}+\mu+d_{r}\right)}, \\
v_{25}=\frac{\gamma \eta(1-q) \rho_{s}}{(\gamma+\mu)\left(\rho_{s}+\mu+d_{s}\right)\left(\rho_{r}+\mu+d_{r}\right)}, v_{55}=\frac{1}{\left(\rho_{r}+\mu+d_{r}\right)}, \\
v_{35}=\frac{(1-v) \delta(1-q) \rho_{s}}{\left(\rho_{s}+\mu+d_{s}\right)(\delta+\mu)\left(\rho_{r}+\mu+d_{r}\right)}, \text { and } v_{45}=\frac{(1-v) \delta}{(\delta+\mu)\left(\rho_{r}+\mu+d_{r}\right)} .
\end{gathered}
$$

The spectral radius of $\mathcal{F} V^{-1}$ is the required effective reproduction number $R_{\text {eff }}$ and obtained as:

$$
\begin{gathered}
R_{e f f}=\max \left\{R_{e f f}(\mathrm{DS}), R_{e f f}(\mathrm{MDR})\right\} \text { Where, } \\
R_{e f f}(\mathrm{DS})=c \omega_{s}\left(\frac{\sigma \psi \mu+(\theta+(1-\psi) \mu)}{\theta+\mu}\right) \frac{(\alpha \varepsilon(1-p) \gamma \eta+\alpha(\gamma+\mu)(1-\varepsilon)(1-p))}{(\alpha+\mu)(\gamma+\mu)\left(\rho_{s}+\mu+d_{s}\right)}, \\
R_{e f f}(\mathrm{MDR})=c \omega_{r}\left(\frac{\sigma \psi \mu+(\theta+(1-\psi) \mu)}{\theta+\mu}\right) \frac{(1-v) \delta}{(\delta+\mu)\left(\rho_{r}+\mu+d_{r}\right)} .
\end{gathered}
$$

\subsection{Local Stability Analysis of the Disease Free Equilibrium Point}

Theorem-3:

The disease free equilibrium point $\left(\frac{\psi \Lambda}{\mu+\theta}, \frac{(\theta+(1-\psi) \mu) \Lambda}{\mu(\mu+\theta)}, 0,0,0,0,0,0,0,0\right)$ of the dynamical system (9)-(18) is locally asymptotically stable if $R_{e f f}(\mathrm{DS})<1$ and $R_{e f f}(\mathrm{MDR})<1$; and $E^{0}$ is unstable otherwise.

Proof: The Jacobean matrix of the dynamical system (9)-(18) at any disease free equilibrium point $E^{0}=\left(\frac{\psi \Lambda}{\mu+\theta}, \frac{(\theta+(1-\psi) \mu) \Lambda}{\mu(\mu+\theta)}, 0,0,0,0,0,0,0,0\right)$ is

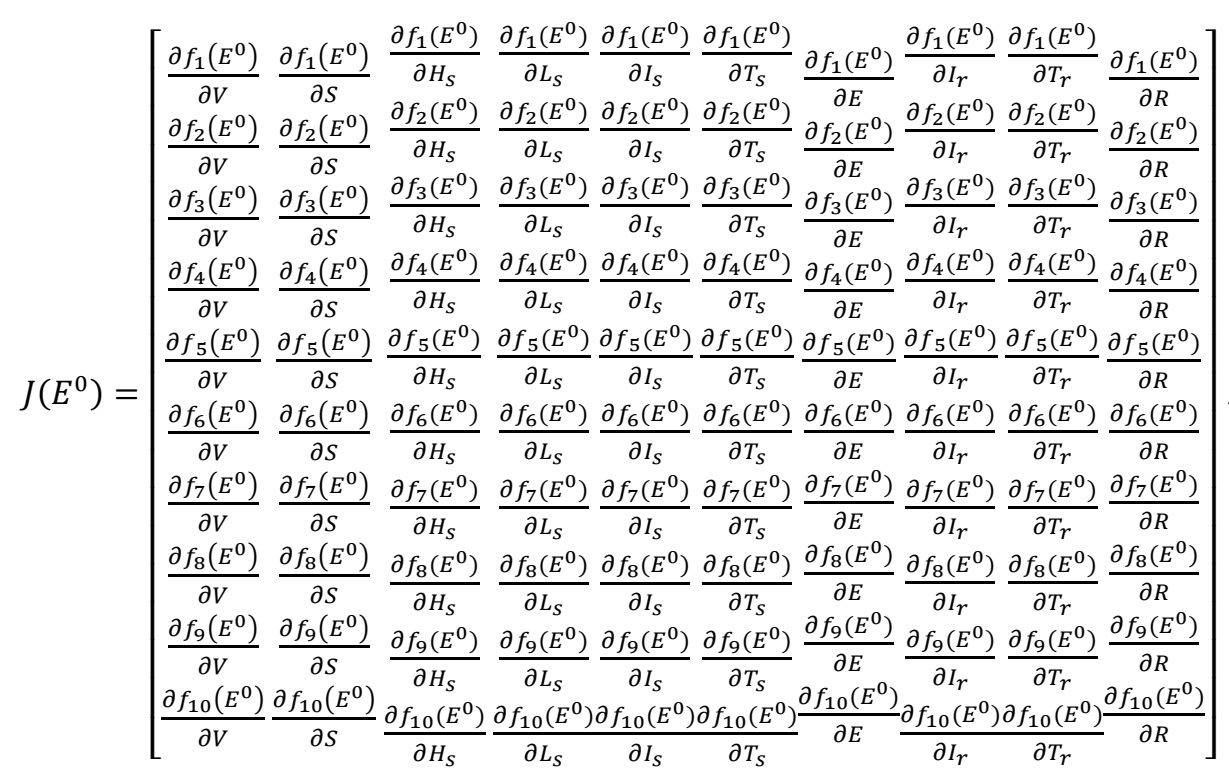




$$
J\left(E^{0}\right)=\left[\begin{array}{cccccccccc}
a_{1} & 0 & 0 & 0 & a_{2} & 0 & 0 & a_{3} & 0 & 0 \\
\theta & -\mu & 0 & 0 & a_{4} & 0 & 0 & a_{5} & 0 & 0 \\
0 & 0 & a_{6} & 0 & a_{7} & 0 & 0 & 0 & 0 & 0 \\
0 & 0 & a_{8} & a_{9} & 0 & 0 & 0 & 0 & 0 & 0 \\
0 & 0 & a_{10} & \gamma \eta & a_{11} & 0 & 0 & 0 & 0 & 0 \\
0 & 0 & \alpha p & 0 & 0 & a_{12} & 0 & 0 & 0 & 0 \\
0 & 0 & 0 & 0 & a_{13} & 0 & a_{14} & a_{15} & 0 & 0 \\
0 & 0 & 0 & 0 & 0 & 0 & a_{16} & a_{17} & 0 & 0 \\
0 & 0 & 0 & 0 & 0 & 0 & v \delta & 0 & a_{18} & 0 \\
0 & 0 & 0 & a_{19} & q \rho_{s} & \phi & 0 & \rho_{r} & \varphi & -\mu
\end{array}\right]
$$

Where

$$
\begin{gathered}
a_{1}=-(\theta+\mu), a_{2}=-\sigma c \omega_{s} V^{0} / N^{0}, \\
a_{3}=-\frac{\sigma c \omega_{r} V^{0}}{N^{0}}, a_{4}=\frac{c \omega_{s} S^{0}}{N^{0}}, a_{5}=-\frac{c \omega_{r} s^{0}}{N^{0}}, a_{6}=-(\alpha+\mu), \\
a_{7}=\frac{c \omega_{s}\left(s^{0}+\sigma V^{0}\right)}{N^{0}}, a_{8}=\alpha \varepsilon(1-p), a_{9}=-(\gamma+\mu), \\
a_{10}=\alpha(1-\varepsilon)(1-p), a_{11}=-\left(\rho_{s}+\mu+d_{s}\right), \\
a_{12}=-(\phi+\mu), a_{13}=(1-q) \rho_{s}, a_{14}=-(\delta+\mu), \\
a_{15}=\frac{c \omega_{r}\left(s^{0}+\sigma V^{0}\right)}{N^{0}}, a_{16}=(1-v) \delta, \\
a_{17}=-\left(\rho_{r}+\mu+d_{r}\right), a_{18}=-(\varphi+\mu), a_{19}=\gamma(1-\eta) .
\end{gathered}
$$

The corresponding characteristic equation is obtained by

$$
\left|\begin{array}{cccccccccc}
a_{1}-\lambda & 0 & 0 & 0 & a_{2} & 0 & 0 & a_{3} & 0 & 0 \\
\theta & -\mu-\lambda & 0 & 0 & a_{4} & 0 & 0 & a_{5} & 0 & 0 \\
0 & 0 & a_{6-\lambda} & 0 & a_{7} & 0 & 0 & 0 & 0 & 0 \\
0 & 0 & a_{8} & a_{9}-\lambda & 0 & 0 & 0 & 0 & 0 & 0 \\
0 & 0 & a_{10} & \gamma \eta & a_{11}-\lambda & 0 & 0 & 0 & 0 & 0 \\
0 & 0 & \alpha p & 0 & 0 & a_{12}-\lambda & 0 & 0 & 0 & 0 \\
0 & 0 & 0 & 0 & a_{13} & 0 & a_{14}-\lambda & a_{15} & 0 & 0 \\
0 & 0 & 0 & 0 & 0 & 0 & a_{16} & a_{17}-\lambda & 0 & 0 \\
0 & 0 & 0 & 0 & 0 & 0 & \nu \delta & 0 & a_{18}-\lambda & 0 \\
0 & 0 & 0 & a_{19} & q \rho_{s} & \phi & 0 & \rho_{r} & \varphi & -\mu-\lambda
\end{array}\right|=0
$$

$$
\begin{gathered}
\text { Or }(\lambda+\mu)^{2}\left(a_{1}-\lambda\right)\left(a_{12}-\lambda\right)\left(a_{18}-\lambda\right)\left[\lambda^{2}-\left(a_{14}+a_{17}\right) \lambda+a_{14} a_{17}-a_{15} a_{16}\right]\left[-\lambda^{3}+\left(a_{6}+a_{9}+a_{11}\right) \lambda^{2}-\left(a_{6} a_{9}+\right.\right. \\
\left.\left.a_{6} a_{11}+a_{9} a_{11}+a_{7} a_{10}\right) \lambda+a_{6} a_{9} a_{11}+\gamma \eta a_{7} a_{8}-a_{7} a_{10} a_{9}\right]=0
\end{gathered}
$$

Thus, the roots of the characteristic equation are $\lambda_{1}=-\mu, \lambda_{2}=-\mu, \lambda_{3}=a_{1}=-(\theta+\mu), \lambda_{4}=a_{12}=-(\phi+\mu)$, $\lambda_{5}=a_{18}=-(\varphi+\mu)$ and/or $\lambda^{2}-\left(a_{14}+a_{17}\right) \lambda+a_{14} a_{17}-a_{15} a_{16}=0$ or $\lambda^{3}-\left(a_{6}+a_{9}+a_{11}\right) \lambda^{2}+\left(a_{6} a_{9}+a_{6} a_{11}+\right.$ $\left.a_{9} a_{11}+a_{7} a_{10}\right) \lambda+a_{6} a_{9} a_{11}-\gamma \eta a_{7} a_{8}+a_{7} a_{10} a_{9}=0$

The Routh-Hurwitz conditions simplifies to $b_{1}>0, b_{2}>0, c_{1}>0, c_{2}>0, c_{3}>0$ and $c_{1} c_{2}>c_{3}$. That is, the necessary conditions for Routh-Hurwitz, $b_{2}>0$ and $c_{3}>0$ is true if $R_{\text {eff }}(\mathrm{MDR})<1$ and $R_{\text {eff }}(\mathrm{MDR})<1$ respectively. The sufficient condition for the Routh-Hurwitz criteria is: $c_{1} c_{2}-c_{3}=\left(3 \mu+\alpha+\gamma+\rho_{s}+d_{s}\right)\left[(\alpha+\mu)(\gamma+\mu)+(\alpha+\mu)\left(\rho_{s}+\mu+d_{s}\right)+\right.$ $\left.(\gamma+\mu)\left(\rho_{s}+\mu+d_{s}\right)\right]-(\alpha+\mu)(\gamma+\mu)\left(\rho_{s}+\mu+d_{s}\right)\left[1-R_{e f f}\right.$ (DS)] $>0$. Thus $c_{1} c_{2}-c_{3}>0$ if and only is $R_{e f f}$ (DS) $<1$. Therefore all of the eigenvalues of the Jacobean matrix have negative real parts when $R_{\text {eff }}(\mathrm{DS})<1$ and $R_{\text {eff }}(M D R)<1$. Hence, the disease free equilibrium $E^{0}$, of the model system (9)-(18) is locally asymptotical stable whenever $R_{e f f}(\mathrm{DS})<1$ and $R_{e f f}(\mathrm{MDR})<1$ and unstable otherwise that is unstable if $R_{\text {eff }}>1$.

\subsection{Global Stability of Diseases Free Equilibrium Point}

Theorem-4:

The diseases free equilibrium point $\left(\frac{\psi \Lambda}{\mu+\theta}, \frac{(\theta+(1-\psi) \mu) \Lambda}{\mu(\mu+\theta)}, 0,0,0,0,0,0,0,0\right)$ of the dynamical system (9) - (18) is globally asymptotically stable in $\Omega$ if $R_{e f f}(\mathrm{DS})<1$ and $R_{\text {eff }}(\mathrm{MDR})<1$, and unstable otherwise.

Proof: We apply a matrix-theoretic method using the Perron eigenvector to prove the global stability of the disease-free equilibrium as in [15]. The dynamical system (9)-(18), the drug sensitive TB disease compartment of is $x_{1}=\left(H_{s}, L_{s}, I_{S}\right)^{T} \epsilon \mathbb{R}^{3}$ 
and non-disease (drug sensitive TB) compartment $y_{1} \in \mathbb{R}^{7}$.

$$
\dot{x_{1}}=\left(F_{1}-V_{1}\right) x_{1}-f_{1}\left(x_{1}, y_{1}\right)
$$

Where, the non-negative matrix $F_{1}$, of the new drug sensitive TB infection terms, and the matrix $V_{1}$, of the transition terms of drug sensitive TB and $f_{1}\left(x_{1}, y_{1}\right)=\left(\lambda_{r} H_{s}, \lambda_{r} L_{s}, 0,\right)^{T}$

$$
V_{1}^{-1} F_{1}=c \omega_{S}\left[\begin{array}{ccc}
0 & 0 & \frac{1}{\alpha+\mu} \\
0 & 0 & \frac{\alpha \varepsilon(1-p)}{(\alpha+\mu)(\gamma+\mu)} \\
0 & 0 & \frac{\alpha \varepsilon(1-p) \gamma \eta+\alpha(1-\varepsilon)(1-p)(\gamma+\mu)}{(\alpha+\mu)(\gamma+\mu)\left(\rho_{s}+\mu+d_{s}\right)}
\end{array}\right] .
$$

Hence, $\lambda_{11}=\lambda_{12}=0$ and $\lambda_{13}=\frac{\alpha \varepsilon(1-p) \gamma \eta+\alpha(1-\varepsilon)(1-p)(\gamma+\mu)}{(\alpha+\mu)(\gamma+\mu)\left(\rho_{s}+\mu+d_{s}\right)}$ are eigenvectors of $V_{1}^{-1} F_{1}$. Let, $\omega_{1}{ }^{T}=\left(u_{1}, u_{2}, u_{3}\right)$ be the left eigenvector of $V_{1}^{-1} F_{1}$ corresponding to $\lambda_{13}=\frac{\alpha \varepsilon(1-p) \gamma \eta+\alpha(1-\varepsilon)(1-p)(\gamma+\mu)}{(\alpha+\mu)(\gamma+\mu)\left(\rho_{s}+\mu+d_{s}\right)}$

Thus, $\omega_{1}{ }^{T} V_{1}^{-1} F_{1}=c \omega_{s}\left(u_{1}, u_{2}, u_{3}\right)\left[\begin{array}{ccc}\Delta & 0 & \frac{1}{\alpha+\mu} \\ 0 & \Delta & \frac{\alpha \varepsilon(1-p)}{(\alpha+\mu)(\gamma+\mu)} \\ 0 & 0 & 0\end{array}\right]$

Where, $\Delta=-\frac{\alpha \varepsilon(1-p) \gamma \eta+\alpha(1-\varepsilon)(1-p)(\gamma+\mu)}{(\alpha+\mu)(\gamma+\mu)\left(\rho_{s}+\mu+d_{s}\right)}$. i.e, $\omega_{1}{ }^{T}=(0,0,1)$ is the left eigenvector of $V_{1}^{-1} F_{1}$ corresponding to the eigenvalue $\lambda_{13}=\frac{\alpha \varepsilon(1-p) \gamma \eta+\alpha(1-\varepsilon)(1-p)(\gamma+\mu)}{(\alpha+\mu)(\gamma+\mu)\left(\rho_{S}+\mu+d_{S}\right)}$.

Lets' define a function $W_{1}\left(H_{S}, L_{S}, I_{S}\right)$ as:

$$
W_{1}=\omega_{1}{ }^{T} V_{1}^{-1} x_{1}=\frac{\alpha \varepsilon(1-p) \gamma \eta+\alpha(1-\varepsilon)(1-p)(\gamma+\mu)}{(\alpha+\mu)(\gamma+\mu)\left(\rho_{s}+\mu+d_{s}\right)} H_{s}+\frac{\gamma \eta}{\gamma+\mu} L_{s}+\frac{1}{\left(\rho_{s}+\mu+d_{s}\right)} I_{s} .
$$

The derivative of $W_{1}$ with respect to time:

$$
\begin{aligned}
\dot{W}_{1}= & \omega_{1}^{T} V_{1}{ }^{-1} \dot{x_{1}},\left[\text { Since, } \dot{x_{1}}=\left(F_{1}-V_{1}\right) x_{1}-f_{1}\left(x_{1}, y_{1}\right)\right] \\
& =\omega_{1}^{T}\left[\left(R_{e f f}(\mathrm{DS})-1\right) x_{1}-V_{1}^{-1} f_{1}\left(x_{1}, y_{1}\right)\right] . \\
& =\omega_{1}^{T}\left(R_{e f f}(\mathrm{DS})-1\right) x_{1}-V_{1}^{-1} f_{1}\left(x_{1}, y_{1}\right) .
\end{aligned}
$$

Since $\omega_{1}{ }^{T}>0, V_{1}{ }^{-1}>0$ and $f_{1}\left(x_{1}, y_{1}\right) \geq 0, \dot{W}_{1}<0$, if $R_{e f f}(D S)<1$.

The drug resistance TB disease compartment of the dynamical system (9)-(18) is $x_{2}=\left(E, I_{r}\right)^{T} \in \mathbb{R}^{2}$ and non-disease (drug resistance TB) compartment $y_{2} \in \mathbb{R}^{8}$.

$$
\dot{x_{2}}=\left(F_{2}-V_{2}\right) x_{2}-f_{2}\left(x_{2}, y_{2}\right)
$$

Where, the non-negative matrix $F_{2}$, of the new drug resistance TB infection terms, and the matrix $V_{2}$, of the transition terms of drug resistance TB and $f_{2}\left(x_{2}, y_{2}\right)=(0,0,)^{T}$.

Therefore,

$$
V_{2}^{-1} F_{2}=\left[\begin{array}{cc}
\frac{1}{(\delta+\mu)} & 0 \\
\frac{\delta(1-v)}{(\delta+\mu)\left(\rho_{r}+\mu+d_{r}\right)} & \frac{1}{\left(\rho_{r}+\mu+d_{r}\right)}
\end{array}\right]\left[\begin{array}{cc}
0 & 0 \\
0 & c \omega_{r}
\end{array}\right]=c \omega_{r}\left[\begin{array}{cc}
0 & \frac{1}{(\delta+\mu)} \\
0 & \frac{\delta(1-v)}{(\delta+\mu)\left(\rho_{r}+\mu+d_{r}\right)}
\end{array}\right] .
$$

Hence, $\lambda_{21}=0$ and $\lambda_{22}=\frac{\delta(1-v)}{(\delta+\mu)\left(\rho_{r}+\mu+d_{r}\right)}$ are eigenvectors of $V_{2}^{-1} F_{2}$. Let, $\omega_{2}{ }^{T}=\left(z_{1}, z_{2},\right)$ be the left eigenvector of $V_{2}^{-1} F_{2}$ corresponding to $\lambda_{22}=\frac{\delta(1-v)}{(\delta+\mu)\left(\rho_{r}+\mu+d_{r}\right)}$

Therefore, $\omega_{2}{ }^{T}=(0,1)$ left eigenvector of $V_{2}^{-1} F_{2}$ corresponding to $\lambda_{22}=\frac{\delta(1-v)}{(\delta+\mu)\left(\rho_{r}+\mu+d_{r}\right)}$.

Lets' define a function $W_{2}\left(E, I_{r}\right)$ as:

$$
W_{2}=\omega_{2}^{T} V_{2}^{-1} x_{2}=\frac{\delta(1-v)}{(\delta+\mu)\left(\rho_{r}+\mu+d_{r}\right)} E+\frac{1}{\left(\rho_{r}+\mu+d_{r}\right)} I_{r}
$$

The derivative $W_{2}$ with respect to time:

$$
\dot{W}_{2}=\omega_{2}{ }^{T} V_{2}{ }^{-1} \dot{x}_{2} \text {, Since, } \dot{x_{2}}=\left(F_{2}-V_{2}\right) x_{2}-f_{2}\left(x_{2}, y_{2}\right)
$$


$=\omega_{2}{ }^{T}\left(R_{\text {eff }}(\mathrm{MDR})-1\right) x_{2}$ and Since $\omega_{2}{ }^{T}>0$, then $\dot{W}_{2}<0$, if $R_{\text {eff }}(\mathrm{MDR})<1$

Now we can define a Lyapunov function $W\left(H_{s}, L_{s}, I_{s}, E, I_{r}\right)$ as:

$$
W\left(H_{s}, L_{s}, I_{s}, E, I_{r}\right)=A_{1} H_{s}+A_{2} L_{s}+A_{3} I_{s}+A_{4} E+A_{5} I_{r}
$$

where, $A_{1}=\frac{\alpha \varepsilon(1-p) \gamma \eta+\alpha(1-\varepsilon)(1-p)(\gamma+\mu)}{(\alpha+\mu)(\gamma+\mu)\left(\rho_{S}+\mu+d_{S}\right)}, A_{2}=\frac{\gamma \eta}{\gamma+\mu}$,

$$
A_{3}=\frac{1}{\left(\rho_{s}+\mu+d_{s}\right)}, A_{4}=\frac{\delta(1-v}{(\delta+\mu)\left(\rho_{r}+\mu+d_{r}\right)}, \text { and } A_{5}=\frac{1}{\left(\rho_{r}+\mu+d_{r}\right)}
$$

That is,

$$
W=W_{1}+W_{2}=\frac{\alpha \varepsilon(1-p) \gamma \eta+\alpha(1-\varepsilon)(1-p)(\gamma+\mu)}{(\alpha+\mu)(\gamma+\mu)\left(\rho_{s}+\mu+d_{s}\right)} H_{s}+\frac{\gamma \eta}{\gamma+\mu} L_{s}+\frac{1}{\left(\rho_{s}+\mu+d_{s}\right)} I_{S}+\frac{\delta(1-v}{(\delta+\mu)\left(\rho_{r}+\mu+d_{r}\right)} E+\frac{1}{\left(\rho_{r}+\mu+d_{r}\right)} I_{r}
$$

Differentiate $W$ with respect to time:

$$
\dot{W}=\frac{\alpha \varepsilon(1-p) \gamma \eta+\alpha(1-\varepsilon)(1-p)(\gamma+\mu)}{(\alpha+\mu)(\gamma+\mu)\left(\rho_{s}+\mu+d_{s}\right)} \dot{H}_{s}+\frac{\gamma \eta}{\gamma+\mu} \dot{L}_{s}+\frac{1}{\left(\rho_{s}+\mu+d_{1}\right)} \dot{I}_{s}+\frac{\delta(1-v}{(\delta+\mu)\left(\rho_{r}+\mu+d_{r}\right)} \dot{E}+\frac{1}{\left(\rho_{r}+\mu+d_{r}\right)} \dot{I}_{r} .
$$

Substituting the derivatives and simplify:

$$
\dot{W}=\left(R_{e f f}(\mathrm{DS})-1\right) I_{s}+\left(R_{e f f}(\mathrm{MDR})-1\right) I_{r}-\lambda_{r}\left(\frac{\alpha \varepsilon(1-p) \gamma \eta+\alpha(1-\varepsilon)(1-p)(\gamma+\mu)}{(\alpha+\mu)(\gamma+\mu)\left(\rho_{s}+\mu+d_{s}\right)} H_{s}+\frac{\gamma \eta}{\gamma+\mu} L_{s}\right) .
$$

Hence, $\quad \dot{W}<0$, if $R_{\text {eff }}(\mathrm{DS})<1$ and $R_{\text {eff }}(\mathrm{MDR})<1$. When $\dot{W}=0$, we must have that $\left(R_{e f f}(\mathrm{DS})-1\right) I_{s}+\left(R_{e f f}(\mathrm{MDR})-1\right) I_{r}=\lambda_{r}\left(\frac{\alpha \varepsilon(1-p) \gamma \eta+\alpha(1-\varepsilon)(1-p)(\gamma+\mu)}{(\alpha+\mu)(\gamma+\mu)\left(\rho_{s}+\mu+d_{s}\right)} H_{s}+\frac{\gamma \eta}{\gamma+\mu} L_{s}\right)$. And $\dot{W}=0$, at the disease free equilibrium point. By LaSalle's invariant principle, every solution to the model equations (9) - (18) with initial conditions in $\Omega$, tends to $E^{0}$ as $t \rightarrow \infty$. Hence, since the region $\Omega$ is positively-invariant, the disease free equilibrium point, $E^{0}$ is globally asymptotically stable in $\Omega$ if $R_{e f f}<1$.

\section{Existence of Endemic Equilibrium Point}

\subsection{The Drug Sensitive TB-strain Only Equilibrium}

This is obtained by setting $\lambda_{r}=0$ and $q=1$ (that treatment of active drug sensitive TB is $100 \%$ effective) in the dynamical system (9)-(18). The drug sensitive TB only equilibrium in terms of the equilibrium value of the force of infection $\lambda_{s}{ }^{*}$ is given as: $E_{1}=\left(V^{*}, S^{*}, H_{s}{ }^{*}, L_{s}{ }^{*}, I_{s}{ }^{*}, T_{s}{ }^{*}, 0,0,0, R^{*}\right)$ where

$$
\begin{aligned}
& V^{*}=\frac{\psi \Lambda}{\sigma \lambda_{s}{ }^{*}+\theta+\mu}, S^{*}=\frac{\Lambda\left[(1-\psi) \sigma \lambda_{s}{ }^{*}+\theta+(1-\psi) \mu\right]}{\left(\lambda_{s}{ }^{*}+\mu\right)\left(\sigma \lambda_{s}{ }^{*}+\theta+\mu\right)}, \\
& H_{s}{ }^{*}=\frac{(\gamma+\mu)\left(\rho_{s}+\mu+d_{s}\right)}{\gamma \eta \alpha \varepsilon(1-p)+\alpha(\gamma+\mu)(1-\varepsilon)(1-p)} I_{s}^{*} \\
& L_{S}{ }^{*}=\frac{\alpha \varepsilon(1-p)\left(\rho_{S}+\mu+d_{S}\right)}{\gamma \eta \alpha \varepsilon(1-p)+\alpha(\gamma+\mu)(1-\varepsilon)(1-p)} I_{S}{ }^{*}, \\
& T_{S}{ }^{*}=\frac{\alpha p(\gamma+\mu)\left(\rho_{S}+\mu+d_{S}\right)}{(\phi+\mu)[\gamma \eta \alpha \varepsilon(1-p)+\alpha(\gamma+\mu)(1-\varepsilon)(1-p)]} I_{S}{ }^{*}, \\
& R^{*}=\frac{1}{\left(\kappa \lambda_{S}{ }^{*}+\mu\right)}\left\{\begin{array}{c}
\rho_{S}+\frac{\gamma(1-\eta) \alpha \varepsilon(1-p)\left(\rho_{S}+\mu+d_{S}\right)}{[\gamma \eta \alpha \varepsilon(1-p)+\alpha(\gamma+\mu)(1-\varepsilon)(1-p)]} \\
+\frac{\phi \alpha p(\gamma+\mu)\left(\rho_{S}+\mu+d_{S}\right)}{(\phi+\mu)[\gamma \eta \alpha \varepsilon(1-p)+\alpha(\gamma+\mu)(1-\varepsilon)(1-p)]}
\end{array}\right\} I_{S}^{*} .
\end{aligned}
$$

\subsubsection{Local Stability of Drug Sensitive TB-strain Only Equilibrium Point, $E_{1}$}

Theorem-5:

The drug sensitive TB only equilibrium $E_{1}$ of the dynamical system (9)-(14), (18) when $q=1$ is locally asymptotically stable if $R_{\text {eff }}(\mathrm{DS})>1>R_{\text {eff }}(\mathrm{MDR})$ and $R^{*}<\frac{\Lambda\left(d_{s}+\mu\right)}{c \omega_{s} \kappa \mu}$.

Proof: The Jacobean matrix of the model system (9)-(14), (18) at equilibrium is given by: 


$$
J\left(E_{1}\right)=\left(\begin{array}{ccccccc}
g_{1} & 0 & 0 & 0 & b_{1} & 0 & 0 \\
\theta & g_{2} & 0 & 0 & b_{2} & 0 & 0 \\
\sigma m^{*} & m^{*} & g_{3} & 0 & z & 0 & \kappa m^{*} \\
0 & 0 & \alpha \varepsilon(1-p) & g_{4} & 0 & 0 & 0 \\
0 & 0 & \alpha(1-\varepsilon)(1-p) & \gamma \eta & g_{5} & 0 & 0 \\
0 & 0 & \alpha p & 0 & 0 & g_{6} & 0 \\
0 & 0 & 0 & \gamma(1-\eta) & \rho_{s} & \phi & g_{7}
\end{array}\right)
$$

Where,

$$
\begin{gathered}
m^{*}=\frac{\sigma-(\sigma \mu+\theta+\mu) a_{1} a_{2} R_{e f f}(D S)}{\sigma a_{1} a_{2} R_{e f f}(D S)}, g_{1}=-\left(\sigma m^{*}+\theta+\mu\right), g_{2}=-\left(m^{*}+\mu\right), g_{3}=-(\alpha+\mu), g_{4}=-(\gamma+\mu), g_{5}=-\left(\rho_{s}+\mu+\right. \\
\left.d_{s}\right), g_{6}=-(\phi+\mu), g_{7}=-\left(\kappa m^{*}+\mu\right), a_{1}=\frac{\mu(\theta+\mu)}{\Lambda[\sigma \psi \mu+(\theta+(1-\psi) \mu)]}, b_{1}=\sigma c \omega_{s} V^{*} / N^{*}, \\
a_{2}=\frac{\Lambda \kappa}{c \omega_{s} \mu}\left\{\begin{array}{c}
\rho_{s}+\frac{\gamma(1-\eta) \alpha \varepsilon(1-p)\left(\rho_{s}+\mu+d_{s}\right)}{[\gamma \eta \alpha \varepsilon(1-p)+\alpha(\gamma+\mu)(1-\varepsilon)(1-p)]}+ \\
\frac{\phi \alpha p(\gamma+\mu)\left(\rho_{s}+\mu+d_{s}\right)}{(\phi+\mu)[\gamma \eta \alpha \varepsilon(1-p)+\alpha(\gamma+\mu)(1-\varepsilon)(1-p)]}
\end{array}\right\} \\
b_{2}=c \omega_{s} V^{*} / N^{*} \text { and } z=\frac{c \omega_{s} \mu}{\Lambda}\left(S^{*}+\sigma V^{*}+\kappa R^{*}\right)
\end{gathered}
$$

The characteristic equation of $J\left(E_{1}\right)$ denoted by $\left|J\left(E_{1}\right)-\lambda I\right|=0$, and given by:

$$
\left|\begin{array}{ccccccc}
g_{1}-\lambda & 0 & 0 & 0 & b_{1} & 0 & 0 \\
\theta & g_{2}-\lambda & 0 & 0 & b_{2} & 0 & 0 \\
\sigma m^{*} & m^{*} & g_{3}-\lambda & 0 & z & 0 & \kappa m^{*} \\
0 & 0 & \alpha \varepsilon(1-p) & g_{4}-\lambda & 0 & 0 & 0 \\
0 & 0 & \alpha(1-\varepsilon)(1-p) & \gamma \eta & g_{5}-\lambda & 0 & 0 \\
0 & 0 & \alpha p & 0 & 0 & g_{6}-\lambda & 0 \\
0 & 0 & 0 & \gamma(1-\eta) & \rho_{s} & \phi & g_{7}-\lambda
\end{array}\right|=0
$$

Now we apply the Gershgorin circle theorem, [16] to determine the sign of the eigenvalues of the characteristic equation $\left|J\left(E_{1}\right)-\lambda I\right|=0$. The matrix $J\left(E_{1}\right)$ is a strictly column diagonally dominant matrix. And also all diagonal elements of $J\left(E_{1}\right)$ are negative. Therefore, using the Gershgorin circle theorem, the radius of the disc less than the magnitude of corresponding element if $R^{*}<\frac{\Lambda\left(d_{S}+\mu\right)}{c \omega_{s} \kappa \mu}$. We can show that all eigenvalues of $J\left(E_{1}\right)$ has negative real part if $R_{e f f}(\mathrm{DS})>1>$ $R_{\text {eff }}(\mathrm{MDR})$ and $R^{*}<\frac{\Lambda\left(d_{S}+\mu\right)}{c \omega_{s} \kappa \mu}$. Hence, the drug resistance TB only equilibrium $E_{1}$ is locally asymptotically stable if $R_{e f f}(\mathrm{DS})>1>R_{e f f}(\mathrm{MDR})$ and $R^{*}<\frac{\Lambda\left(d_{s}+\mu\right)}{c \omega_{s} \kappa \mu}$.

\subsubsection{Global Stability of Drug Sensitive TB only Endemic Equilibrium Point, $E_{1}$}

Theorem-6:

The drug sensitive TB only equilibrium $E_{1}$ of Model (9)-(14), (18) is globally asymptotically stable if $q=1, R_{e f f}$ (DS) $>$ $1>R_{\text {eff }}(\mathrm{MDR})$.

Proof: We use a graph-theoretic method as in [15] to construct a Lyapunov function.

We define functions:

$$
\begin{gathered}
D_{1}=V-V^{*}-V^{*} \ln \frac{V}{V^{*}}, D_{2}=S-S^{*}-S^{*} \ln \frac{S}{S^{*}}, \\
D_{3}=H_{s}-H_{s}{ }^{*}-H_{s}{ }^{*} \ln \frac{H_{S}}{H_{s}{ }^{*}}, D_{4}=L_{s}-L_{s}{ }^{*}-L_{s}{ }^{*} \ln \frac{L_{S}}{L_{s}{ }^{*}} \\
D_{5}=T_{s}-T_{s}{ }^{*}-T_{s}{ }^{*} \ln \frac{T_{S}}{T_{s}{ }^{*}}, D_{6}=I_{S}-I_{s}{ }^{*}-I_{s}{ }^{*} \ln \frac{I_{S}}{I_{S}{ }^{*}}, \\
D_{7}=R-R^{*}-R^{*} \ln \frac{R}{R^{*}}
\end{gathered}
$$

Differentiating the functions $D_{i}$ for $i=1, \ldots, 7$ with respect to time, and using the inequality $1-x+\ln x \leq 0$, for all $x>0$ and the values at the endemic equilibrium point $E_{1}$ that:

$$
\begin{gathered}
D_{1}^{\prime} \leq \sigma \lambda_{s}{ }^{*} V^{*}\left(-\ln \frac{I_{s}}{I_{s}{ }^{*}}+\ln \frac{V^{*}}{V}-\frac{V^{*}}{V}+\frac{I_{s}}{I_{s}{ }^{*}}\right)=a_{15} G_{15} \\
D_{2}^{\prime} \leq \theta V^{*}\left(\frac{S^{*}}{S}-2-\ln \frac{V S^{*}}{V^{*} S}+\frac{V}{V^{*}}\right)+\frac{c \omega_{s}}{N^{*}} S^{*} I_{s}{ }^{*}\left(-\ln \frac{I_{S}}{I_{S}{ }^{*}}+\ln \frac{S^{*}}{S}-\frac{S^{*}}{S}+\frac{I_{s}}{I_{S}{ }^{*}}\right)=a_{21} G_{21}+a_{25} G_{25}
\end{gathered}
$$




$$
\begin{aligned}
& D_{3}^{\prime} \leq \lambda_{s}{ }^{*} S^{*}\left(\frac{I_{S} S}{I_{s}{ }^{*} S^{*}}-\frac{I_{S}}{I_{s}{ }^{*}}-\ln \frac{S}{S^{*}}\right)+\sigma V^{*} \lambda_{s}{ }^{*}\left(\frac{I_{S} V}{V^{*} I_{S}{ }^{*}}-\frac{I_{S}}{I_{s}{ }^{*}}-\ln \frac{V}{V^{*}}\right)+\kappa R^{*} \lambda_{s}{ }^{*}\left(\frac{I_{S} R}{R^{*} I_{s}{ }^{*}}-\frac{I_{s}}{I_{s}{ }^{*}}-\ln \frac{R}{R^{*}}\right) \\
& +\left(S^{*}+\sigma V^{*}+\kappa R^{*}\right) \lambda_{s}{ }^{*}\left(\frac{I_{s}}{I_{s}{ }^{*}}-\frac{H_{s}}{H_{s}{ }^{*}}+\ln \frac{H_{s}}{H_{s}{ }^{*}}-\ln \frac{I_{s}}{I_{s}{ }^{*}}\right) \\
& =: a_{32} G_{32}+a_{31} G_{31}+a_{37} G_{37}+a_{35} G_{35} \\
& D_{4}^{\prime} \leq \alpha \varepsilon(1-p) H_{s}{ }^{*}\left(-\frac{L_{s}}{L_{s}{ }^{*}}+\ln \frac{L_{s}}{L_{s}{ }^{*}}-\ln \frac{H_{s}}{H_{s}{ }^{*}}+\frac{H_{s}}{H_{s}{ }^{*}}\right)=a_{43} G_{43} \\
& D_{5}^{\prime} \leq \gamma \eta L_{s}{ }^{*}\left(-\frac{I_{S}}{I_{S}{ }^{*}}+\ln \frac{I_{S}}{I_{s}{ }^{*}}-\ln \frac{L_{S}}{L_{S}{ }^{*}}+\frac{L_{S}}{L_{s}{ }^{*}}\right)+\alpha(1-\varepsilon)(1-p) H_{S}{ }^{*}\left(-\frac{I_{S}}{I_{S}{ }^{*}}+\ln \frac{I_{S}}{I_{S}{ }^{*}}-\frac{H_{S}}{H_{S}{ }^{*}}+\frac{H_{S}}{H_{S}{ }^{*}}\right) \\
& =: a_{54} G_{54}+a_{53} G_{53} \\
& D_{6}^{\prime} \leq(\phi+\mu) T_{s}{ }^{*}\left(\frac{H_{s}}{H_{s}{ }^{*}}-\ln \frac{H_{S}}{H_{s}{ }^{*}}-\frac{T_{s}}{T_{s}{ }^{*}}+\ln \frac{T_{s}{ }^{*}}{T_{s}}\right)=: a_{63} G_{63} \\
& D_{7}^{\prime} \leq \rho_{S} I_{S}{ }^{*}\left(\frac{I_{S}}{I_{S}{ }^{*}}-\ln \frac{I_{S}}{I_{S}{ }^{*}}+\ln \frac{R}{R^{*}}-\frac{R}{R^{*}}\right)+\gamma(1-\eta) L_{S}{ }^{*}\left(-\frac{R}{R^{*}}+\ln \frac{R}{R^{*}}-\ln \frac{L_{S}}{L_{S}{ }^{*}}+\frac{L_{S}}{L_{S}{ }^{*}}\right)+\phi T_{S}{ }^{*}\left(-\frac{R}{R^{*}}+\ln \frac{R}{R^{*}}-\ln \frac{T_{S}}{T_{S}{ }^{*}}+\frac{T_{S}}{T_{S}{ }^{*}}\right)+ \\
& \kappa I_{S}^{*} R^{*}\left(\frac{R}{R^{*}}-2-\ln \frac{R}{R^{*}} \frac{I_{S}}{I_{S}{ }^{*}}+\frac{I_{S}}{I_{S}^{*}}\right) \\
& =: a_{74} G_{74}+a_{76} G_{76}+a_{75 a} G_{75 a}+a_{75 b} G_{75 b} .
\end{aligned}
$$

Where,

$$
\begin{gathered}
a_{15}=a_{31}=\sigma V^{*} \lambda_{s}{ }^{*}, a_{21}=\theta, a_{25}=a_{32}=S^{*} \lambda_{s}{ }^{*}, a_{37}=\kappa R^{*} \lambda_{s}{ }^{*}, a_{35}=\left(S^{*}+\sigma V^{*}+\kappa R^{*}\right) \lambda_{s}{ }^{*}, \\
a_{43}=\alpha \varepsilon(1-p) H_{s}{ }^{*}, a_{54}=\gamma \eta L_{s}{ }^{*}, a_{63}=(\phi+\mu) T_{s}{ }^{*}, \\
a_{53}=\alpha(1-\varepsilon)(1-p) H_{s}{ }^{*}, a_{74}=\rho_{s} I_{s}{ }^{*}, \\
a_{76}=\gamma(1-\eta) L_{s}^{*}, a_{75 a}=\phi T_{s}^{*}, a_{75 b}=\kappa \lambda_{s}{ }^{*} R^{*} \text { and all other } a_{i j}=0 .
\end{gathered}
$$

The associated weighted digraph G (A) has seven vertices. Along each cycle:

$$
\begin{aligned}
& G_{35}+G_{53}=\left(\frac{I_{S}}{I_{s}{ }^{*}}-\frac{H_{S}}{H_{S}{ }^{*}}+\ln \frac{H_{S}}{H_{S}{ }^{*}}-\ln \frac{I_{S}}{I_{S}{ }^{*}}\right)+\left(-\frac{I_{S}}{I_{S}{ }^{*}}+\ln \frac{I_{S}}{I_{S}{ }^{*}}-\frac{H_{S}}{H_{S}{ }^{*}}+\frac{H_{S}}{H_{S}{ }^{*}}\right)=0 \text { and } \\
& G_{35}+G_{43}+G_{54}=\left(\frac{I_{S}}{I_{s}{ }^{*}}-\frac{H_{S}}{H_{S}{ }^{*}}+\ln \frac{H_{S}}{H_{S}{ }^{*}}-\ln \frac{I_{S}}{I_{s}{ }^{*}}\right)+\left(-\frac{L_{S}}{L_{s}{ }^{*}}+\ln \frac{L_{S}}{L_{S}{ }^{*}}-\ln \frac{H_{S}}{H_{S}{ }^{*}}+\frac{H_{S}}{H_{S}{ }^{*}}\right)+\left(-\frac{I_{S}}{I_{S}{ }^{*}}+\ln \frac{I_{S}}{I_{S}{ }^{*}}-\ln \frac{L_{S}}{L_{S}{ }^{*}}+\frac{L_{S}}{L_{S}{ }^{*}}\right)=0 \text {. And the }
\end{aligned}
$$
other cycles $\sum G_{i j} \leq 0$, if $\frac{V}{V^{*}}, \frac{S}{S^{*}}, \frac{R}{R^{*}} \leq 1$ and $\frac{H_{S}}{H_{s}{ }^{*}} \leq \frac{I_{s}}{I_{s}{ }^{*}}$. By Proposition 1.3 of [15], there exists $c_{i}>0, i=1, \ldots, 7$ such that $D=\sum_{i=1}^{6} c_{i} D_{i}$ is a Lyapunov function for equations (9)-(14),(18). The relations between $c_{i}$ 's can be derived from Theorems 3.3 and 3.4 of [15] such that:

$$
\begin{gathered}
a_{32}>0 \text { and } d^{+}(2)=1 \text { implies } c_{3}=c_{2} \frac{\left(a_{21}+a_{25}\right)}{a_{32}} . \\
a_{15}>0 \text { and } d^{-}(1)=1 \text { implies } c_{1}=c_{2}\left(\frac{a_{21} a_{32}+a_{31}\left(a_{21}+a_{25}\right)}{a_{32} a_{15}}\right) . \\
a_{43}>0 \text { and } d^{-}(4)=1 \text { implies } c_{4}=c_{5} \frac{a_{54}}{a_{43}} . \\
a_{37}>0 \text { and } d^{+}(7)=1 \text { implies } c_{7}=c_{2} \frac{a_{37\left(a_{21}+a_{25}\right)}}{a_{32}\left(a_{74}+a_{75 a}+a_{75 b}+a_{76}\right)} . \\
a_{76}>0 \text { and } d^{+}(6)=1 \text { implies } c_{6}=c_{2} \frac{a_{76} a_{37}\left(a_{21}+a_{25}\right)}{a_{32}\left(a_{65}+a_{63}\right)\left(a_{74}+a_{75 a}+a_{75 b}+a_{76}\right)} .
\end{gathered}
$$

Therefore, $D=c_{1} D_{1}+c_{2} D_{2}+c_{3} D_{3}+c_{4} D_{4}+c_{5} D_{5}+c_{6} D_{6}+c_{7} D_{7}$ is a Lyapunov function for (9)- (14), (18). Therefore, $E_{1}$ is globally asymptotically stable in the interior of $\Omega$ when $R_{e f f}(\mathrm{DS})>1>R_{\text {eff }}(\mathrm{MDR})$.

\subsection{The Drug Resistant TB Strain only Equilibrium Point, $E_{2}$}

This equilibrium solution is obtained by setting $\lambda_{s}=0$ in equations (9)-(18) of the model. The drug resistant TB expressed only in terms of the equilibrium value of the force of infection $\lambda_{r}{ }^{*}$ is given by: 


$$
E_{2}=\left(V^{*}, S^{*}, 0,0,0,0, E^{*}, I_{r}^{*}, T_{r}^{*}, R^{*}\right)
$$

Where, $V^{*}=\frac{\psi \Lambda}{\sigma \lambda_{r}{ }^{*}+\theta+\mu}, S^{*}=\frac{\Lambda\left[(1-\psi) \sigma \lambda_{r}{ }^{*}+\theta+(1-\psi) \mu\right]}{\left(\lambda_{r}{ }^{*}+\mu\right)\left[\sigma \lambda_{r}{ }^{*}+\theta+\mu\right]}, E^{*}=\frac{\left(\rho_{r}+\mu+d_{r}\right)}{(1-v) \delta} I_{r}{ }^{*}, T_{r}{ }^{*}=\frac{v \delta\left(\rho_{r}+\mu+d_{r}\right)}{(\varphi+\mu)(1-v) \delta} I_{r}{ }^{*}$ and $R^{*}=\frac{\rho_{r}(\varphi+\mu)(1-v) \delta+\varphi v \delta\left(\rho_{r}+\mu+d_{r}\right)}{\left(\kappa \lambda_{r}{ }^{*}+\mu\right)(\varphi+\mu)(1-v) \delta} I_{r}{ }^{*}$.

\subsubsection{Local stability of the Drug Resistant TB Strain only Equilibrium Point, $E_{2}$}

Theorem-7:

The drug resistance TB only equilibrium $E_{2}$ of Model (9), (10), (15)-(18) is locally asymptotically stable if $R_{e f f}(\mathrm{DS})<$ $1<R_{\text {eff }}(\mathrm{MDR})$ and $R^{*}<\frac{\Lambda\left(\mu+d_{r}\right)}{c \omega_{r} \kappa \mu}$.

Proof: The Jacobean matrix of the dynamical system (9),(10), (15)-(18) at equilibrium $E_{2}$ is given by:

$$
J\left(E_{2}\right)=\left(\begin{array}{cccccc}
f_{1} & 0 & 0 & a_{1} & 0 & 0 \\
\theta & f_{2} & 0 & a_{2} & 0 & 0 \\
\sigma n^{*} & n^{*} & f_{3} & y & 0 & \kappa n^{*} \\
0 & 0 & (1-v) \delta & f_{4} & 0 & 0 \\
0 & 0 & v \delta & 0 & f_{5} & 0 \\
0 & 0 & 0 & \rho_{r} \varphi & f_{6}
\end{array}\right)
$$

Where, $n^{*}=\frac{\mu \sigma(1+\kappa)+\kappa(\theta+\mu)-R_{e f f}(M D R) e_{1} \kappa\left[e_{2}(\mu \sigma+\theta+\mu)+\sigma\right]}{\sigma \kappa\left(R_{e f f}(M D R) e_{1} e_{2}-1\right)}, e_{1}=\frac{\mu(\theta+\mu)}{[\sigma \psi \mu+(\theta+(1-\psi) \mu)]}, e_{2}=\frac{\left[\rho_{r}(\varphi+\mu)(1-v) \delta+\varphi v \delta\left(\rho_{r}+\mu+d_{r}\right)\right]}{c \omega_{r} \mu(\varphi+\mu)(1-v) \delta}, f_{1}=$ $-\left(\sigma n^{*}+\theta+\mu\right), g_{2}=-\left(n^{*}+\mu\right), f_{3}=-(\delta+\mu), f_{4}=-\left(\rho_{r}+\mu+d_{r}\right), f_{5}=-(\varphi+\mu), f_{6}=-\left(\kappa n^{*}+\mu\right), a_{1}=-\frac{\sigma c \omega_{r} V^{*}}{N^{*}}$, $a_{2}=-\frac{c \omega_{r} S^{*}}{N^{*}}$ and $y=\frac{c \omega_{r} \mu}{\Lambda}\left(S^{*}+\sigma V^{*}+\kappa R^{*}\right)$.

The characteristic equation of the matrix $J\left(E_{2}\right)$ is given by $\left|J\left(E_{2}\right)-\lambda I\right|=0$ :

$$
\left|\begin{array}{cccccc}
f_{1}-\lambda & 0 & 0 & a_{1} & 0 & 0 \\
\theta & f_{2}-\lambda & 0 & a_{2} & 0 & 0 \\
\sigma n^{*} & n^{*} & f_{3}-\lambda & y & 0 & \kappa n^{*} \\
0 & 0 & (1-v) \delta & f_{4}-\lambda & 0 & 0 \\
0 & 0 & v \delta & 0 & f_{5}-\lambda & 0 \\
0 & 0 & 0 & \rho_{r} & \varphi & f_{6}-\lambda
\end{array}\right|=0
$$

Now we apply the Gershgorin circle theorem, [16] to determine the sign of the eigenvalues of the characteristic equation $\left|J\left(E_{2}\right)-\lambda I\right|=0$. In the matrix $J\left(E_{2}\right),\left|c_{i i}\right|>\sum_{\substack{i=1 \\ i \neq j}}^{6} c_{i j}$, for $j=1, \ldots, 6$, and $R^{*}<\frac{\Lambda\left(\mu+d_{r}\right)}{c \omega_{r} \kappa \mu}$ then $J\left(E_{2}\right)$ is a strictly column diagonally dominant matrix and also all diagonal elements of $J\left(E_{2}\right)$ are negative. Hence, all eigenvalues of $J\left(E_{2}\right)$ has negative real part if $R_{e f f}(\mathrm{DS})<1<R_{e f f}(\mathrm{MDR})$ and $R^{*}<\frac{\Lambda\left(\mu+d_{r}\right)}{c \omega_{r} \kappa \mu}$. Therefore, the drug resistance TB only equilibrium $E_{2}$ is locally asymptotically stable if $R_{e f f}(\mathrm{DS})<1<R_{e f f}(\mathrm{MDR})$ and $R^{*}<\frac{\Lambda\left(\mu+d_{r}\right)}{c \omega_{r} \kappa \mu}$.

\subsubsection{Global Stability of Drug Resistant TB only Endemic Equilibrium Point}

Theorem-8:

The drug resistance TB only equilibrium $E_{2}$ of Model (9), (10), (15)-(18) is globally asymptotically stable if $R_{e f f}$ (DS) $<$ $1<R_{\text {eff }}(\mathrm{MDR})$.

Proof: We use a graph-theoretic method as in [15] to construct a lyapunov function.

We define functions:

$$
\begin{gathered}
Q_{1}=V-V^{*}-V^{*} \ln \frac{V}{V^{*}}, Q_{2}=S-S^{*}-S^{*} \ln \frac{S}{S^{*},} \\
Q_{3}=E-E^{*}-E^{*} \ln \frac{E}{E^{*}}, Q_{4}=I_{r}-I_{r}{ }^{*}-I_{r}{ }^{*} \ln \frac{I_{r}}{I_{r}{ }^{*}}, \\
Q_{5}=T_{r}-T_{r}{ }^{*}-T_{r}{ }^{*} \ln \frac{T_{r}}{T_{r}{ }^{*}}, Q_{6}=R-R^{*}-R^{*} \ln \frac{R}{R^{*}}
\end{gathered}
$$

Where $E_{2}=\left(V^{*}, S^{*}, E^{*}, T_{r}{ }^{*}, I_{r}{ }^{*}, R^{*}\right)$ is the drug resistant TB only endemic equilibrium.

Differentiating the functions $Q_{i}$ for $i=1, \ldots, 6$ with respect to time, and using the inequality $1-x+\ln x \leq 0$, for all $x>0$ and the values at the endemic equilibrium point $E_{2}$ that:

$$
\begin{gathered}
Q_{1} \leq \leq \sigma V^{*} \lambda_{r}{ }^{*}\left(\frac{I_{r}}{I_{r}{ }^{*}}-\ln \frac{V^{*}}{V}-\frac{I_{r} V}{I_{r}{ }^{*} V^{*}}\right)=: b_{14} Q_{14} . \\
Q_{2} \leq \theta V^{*}\left(-2-\ln \frac{V}{V^{*}}-\ln \frac{S^{*}}{S}+\frac{S^{*}}{S}+\frac{V}{V^{*}}\right)=: b_{21} Q_{21} .
\end{gathered}
$$




$$
\begin{aligned}
& Q_{3} \leq\left(\frac{I_{r}}{I_{r}{ }^{*}}-\frac{E}{E^{*}}-\ln \frac{I_{r}}{I_{r}{ }^{*}}+\ln \frac{E}{E^{*}}\right)+\lambda_{r}{ }^{*} S^{*}\left(\frac{I_{r} S}{I_{r}{ }^{*} S^{*}}-\frac{I_{r}}{I_{r}{ }^{*}}-\ln \frac{S}{S^{*}}\right)+\sigma \lambda_{r}{ }^{*} V^{*}\left(\frac{I_{r} V}{I_{r}{ }^{*} V^{*}}-\frac{I_{r}}{I_{r}{ }^{*}}-\ln \frac{V}{V^{*}}\right)+\kappa \lambda_{r}^{*} R^{*}\left(\frac{I_{r} R}{I_{r}{ }^{*} R^{*}}-\frac{I_{r}}{I_{r}{ }^{*}}-\ln \frac{R}{R^{*}}\right) . \\
& =: b_{34} Q_{34}+b_{31} Q_{31}+b_{32} Q_{32}+b_{36} Q_{36} \text {. } \\
& Q_{4} \leq(1-v) \delta E^{*}\left(\frac{E}{E^{*}}-\frac{I_{r}}{I_{r}{ }^{*}}-\ln \frac{E}{E^{*}}+\ln \frac{I_{r}}{I_{r}{ }^{*}}\right)=: b_{43} Q_{43} \text {. } \\
& Q_{5} \leq v \delta E^{*}\left(\frac{E}{E^{*}}-\frac{T_{r}}{T_{r}{ }^{*}}-\ln \frac{E}{E^{*}}+\ln \frac{T_{r}}{T_{r}{ }^{*}}\right)=: b_{53} Q_{53} . \\
& Q_{6} \leq \rho_{r} I_{r}{ }^{*}\left(\frac{I_{r}}{I_{r}{ }^{*}}-\ln \frac{I_{r}}{I_{r}{ }^{*}}+\ln \frac{R}{R^{*}}-\frac{R}{R^{*}}\right)+\varphi T_{r}^{*}\left(\frac{T_{r}}{T_{r}{ }^{*}}-\ln \frac{T_{r}}{T_{r}{ }^{*}}+\ln \frac{R}{R^{*}}-\frac{R}{R^{*}}\right)+\kappa \lambda_{r}{ }^{*} R^{*}\left(-\ln \frac{R}{R^{*}}-\ln \frac{I_{r}}{I_{r}{ }^{*}}-2+\frac{R}{R^{*}}+\frac{I_{r}}{I_{r}{ }^{*}}\right) . \\
& =: b_{64} Q_{64}+b_{65 a} Q_{65 a}+b_{65 b} Q_{65 b}
\end{aligned}
$$

Where, $b_{14}=b_{32}=\sigma \lambda_{r}^{*} V^{*}, b_{21}=\theta V^{*}$, $b_{31}=\lambda_{r}{ }^{*} S^{*}, b_{34}=\left(\lambda_{r}{ }^{*} S^{*}+\sigma \lambda_{r}{ }^{*} V^{*}+\kappa \lambda_{r}{ }^{*} R^{*}\right)$,

$b_{36}=b_{65 b}=\kappa \lambda_{r}{ }^{*} R^{*}, b_{43}=(1-v) \delta E^{*}, b_{53}=v \delta E^{*}, b_{64}=\rho_{r} I_{r}{ }^{*}, b_{65 a}=\varphi T_{r}{ }^{*}$, and all other $b_{i j}=0$.

The associated weighted digraph $\mathrm{G}(\mathrm{Q})$ has six vertices. Along each cycle $G_{34}+G_{43}=\left(\frac{I_{r}}{I_{r}{ }^{*}}-\frac{E}{E^{*}}-\ln \frac{I_{r}}{I_{r}{ }^{*}}+\ln \frac{E}{E^{*}}\right)+$ $\left(\frac{E}{E^{*}}-\frac{I_{r}}{I_{r}{ }^{*}}-\ln \frac{E}{E^{*}}+\ln \frac{I_{r}}{I_{r}{ }^{*}}\right)=0$ and the other cycles $\sum G_{i j} \leq 0$, if $\frac{V}{V^{*}}, \frac{S}{S^{*}}, \frac{R}{R^{*}} \leq 1$ and $\frac{E}{E^{*}} \leq \frac{I_{r}}{I_{r}{ }^{*}}$. By Proposition 1.3 of [15], there exists $c_{i}>0, i=1, \ldots, 6$ such that $Q=\sum_{i=1}^{6} c_{i} Q_{i}$ is a Lyapunov function for equations (9), (10), (15)-(18). The relations between $c_{i}$ 's can be derived from Theorems 3.3 and 3.4 of [15] such that:

$$
\begin{gathered}
b_{21}>0 \text { and } d^{+}(1)=1 \text { implies } c_{2}=c_{1} \frac{b_{14}}{b_{21}} \cdot b_{32}>0 \text { and } d^{+}(2)=1 \text { implies } c_{3}=c_{1} \frac{b_{14}}{b_{32}} . \\
b_{36}>0 \text { and } d^{+}(6)=1 \text { implies } c_{6}=c_{1} \frac{b_{14}}{b_{32}} \frac{\left(b_{34}+b_{31}+b_{32}+b_{36}\right)}{b_{53}} \frac{b_{36}}{\left(b_{64}+b_{65 a}+b_{65 b}\right)} \cdot b_{53}>0 \text { and } d^{-}(5)=1 \text { implies } c_{5}= \\
c_{1} \frac{\left(b_{65}+b_{65 a}\right)}{b_{53}} \frac{b_{14}}{b_{32}} \frac{\left(b_{34}+b_{31}+b_{32}+b_{36}\right)}{b_{53}} \frac{b_{36}}{\left(b_{64}+b_{65 a}+b_{65 b}\right)} \cdot b_{43}>0 \text { and } d^{-}(4)=1 \text { implies }
\end{gathered}
$$$$
c_{4}=c_{1} \frac{1}{b_{43}}\left(b_{14}+\frac{b_{14} b_{34}}{b_{32}}+\frac{b_{14}\left(b_{34}+b_{31}+b_{32}+b_{36}\right)}{b_{32} b_{53}} \frac{b_{36} b_{64}}{\left(b_{64}+b_{65}+b_{65 b}\right)}\right) \text {. }
$$

Therefore, $Q=c_{1} Q_{1}+c_{2} Q_{2}+c_{3} Q_{3}+c_{4} Q_{4}+c_{5} Q_{5}+c_{6} Q_{6}$ is a Lyapunov function for (9), (10), (15)-(18). Therefore, $E_{2}$ is globally asymptotically stable in the interior of $\Omega$ when $R_{e f f}(\mathrm{MDR})>1$.

\subsection{Endemic Equilibrium Point where Both TB Strains Co-exist, $E_{3}$}

The endemic equilibrium where both TB strains co-exist is given as: $E_{3}=\left(V^{*}, S^{*}, H_{s}{ }^{*}, L_{s}{ }^{*}, I_{s}{ }^{*}, T_{s}{ }^{*}, E^{*}, I_{r}{ }^{*}, T_{r}{ }^{*}, R^{*}\right)$,

$$
\begin{aligned}
& \text { Where, } V^{*}=\frac{\psi \Lambda}{\sigma\left(\lambda_{s}{ }^{*}+\lambda_{r}{ }^{*}\right)+\theta+\mu}, S^{*}=\frac{\Lambda\left[(1-\psi) \sigma\left(\lambda_{s}{ }^{*}+\lambda_{r}{ }^{*}\right)+\theta+(1-\psi) \mu\right]}{\left(\lambda_{s}{ }^{*}+\lambda_{r}{ }^{*}+\mu\right)\left[\sigma\left(\lambda_{s}{ }^{*}+\lambda_{r}{ }^{*}\right)+\theta+\mu\right]}, H_{S}{ }^{*}=\frac{\left(\lambda_{r}{ }^{*}+\gamma+\mu\right)\left(\rho_{s}+\mu+d_{s}\right)}{\gamma \eta \alpha \varepsilon(1-p)+\left(\lambda_{r}{ }^{*}+\gamma+\mu\right) \alpha(1-\varepsilon)(1-p)} I_{S}{ }^{*} \text {, } \\
& L_{S}{ }^{*}=\frac{\alpha \varepsilon(1-p)\left(\lambda_{r}{ }^{*}+\gamma+\mu\right)\left(\rho_{S}+\mu+d_{S}\right)}{\gamma \eta \alpha \varepsilon(1-p)+\left(\lambda_{r}{ }^{*}+\gamma+\mu\right) \alpha(1-\varepsilon)(1-p)} I_{S}{ }^{*}, T_{S}{ }^{*}=\frac{\alpha p\left(\lambda_{r}{ }^{*}+\gamma+\mu\right)\left(\rho_{S}+\mu+d_{S}\right)}{(\phi+\mu)\left[\gamma \eta \alpha \varepsilon(1-p)+\left(\lambda_{r}{ }^{*}+\gamma+\mu\right) \alpha(1-\varepsilon)(1-p)\right]} I_{S}{ }^{*}, \\
& E^{*}=\frac{\lambda_{r}{ }^{*}\left(S^{*}+H_{S}{ }^{*}+L_{S}{ }^{*}+\sigma V^{*}+\kappa R^{*}\right)+(1-q) \rho_{S} I_{S}{ }^{*}}{(\delta+\mu)}, T_{r}{ }^{*}=\frac{v\left[\left(\rho_{r}+\mu+d_{r}\right) I_{r}{ }^{*}\right]}{(\varphi+\mu)(1-v)}, R^{*}=\frac{1}{\left(\kappa\left(\lambda_{s}{ }^{*}+\lambda_{r}{ }^{*}\right)+\mu\right)}\left\{\rho_{S}+\frac{\gamma(1-\eta) \alpha \varepsilon(1-p)\left(\rho_{S}+\mu+d_{S}\right)\left(\lambda_{r}{ }^{*}+\gamma+\mu\right)}{\gamma \eta \alpha \varepsilon(1-p)+\left(\lambda_{r}{ }^{*}+\gamma+\mu\right) \alpha(1-\varepsilon)(1-p)}+\right. \\
& \left.\frac{\phi \alpha p\left(\rho_{S}+\mu+d_{S}\right)\left(\lambda_{r}{ }^{*}+\gamma+\mu\right)}{(\phi+\mu)\left[\gamma \eta \alpha \varepsilon(1-p)+\left(\lambda_{r}{ }^{*}+\gamma+\mu\right) \alpha(1-\varepsilon)(1-p)\right]}\right\} I_{S}^{*}+\frac{1}{\left(\kappa\left(\lambda_{S}{ }^{*}+\lambda_{r}{ }^{*}\right)+\mu\right)}\left\{\rho_{r}+\frac{\varphi v\left[\left(\rho_{r}+\mu+d_{r}\right)\right]}{(\varphi+\mu)(1-v)}\right\} I_{r}^{*} \text {. }
\end{aligned}
$$

\subsubsection{Local Stability of Endemic Equilibrium Point where Both TB Strains Co-exist, $E_{3}$}

Theorem-9:

The endemic equilibrium point where both TB strains co-exist $E_{3}$ of the dynamical system (9)-(18) is locally asymptotically stable if $R_{e f f}(\mathrm{DS})>1, R_{e f f}(\mathrm{MDR})>1$ and $R^{*}<\min \left\{\frac{\Lambda\left(\mu+d_{s}\right)}{c \omega_{s} \mu \kappa}, \frac{\Lambda\left(\mu+d_{r}\right)}{c \omega_{r} \mu \kappa}\right\}$.

Proof: The Jacobian matrix of the dynamical system (9)-(18) at endemic equilibrium where both TB strains co-exist $E_{3}$ is given by:

$$
J\left(E_{3}\right)=\left(\begin{array}{cccccccccc}
d_{1} & 0 & 0 & 0 & e_{1} & 0 & 0 & h_{1} & 0 & 0 \\
\theta & d_{2} & 0 & 0 & e_{2} & 0 & 0 & h_{2} & 0 & 0 \\
\sigma \lambda_{s}{ }^{*} & \lambda_{s}{ }^{*} & d_{3} & 0 & e_{3} & 0 & 0 & h_{3} & 0 & \kappa \lambda_{s}{ }^{*} \\
0 & 0 & h_{6} & d_{4} & 0 & 0 & 0 & h_{4} & 0 & 0 \\
0 & 0 & h_{7} & \gamma \eta & d_{5} & 0 & 0 & 0 & 0 & 0 \\
0 & 0 & \alpha p & 0 & 0 & d_{6} & 0 & 0 & 0 & 0 \\
\sigma \lambda_{r}{ }^{*} & \lambda_{r}{ }^{*} & \lambda_{r}{ }^{*} & \lambda_{r}{ }^{*} & h_{8} & 0 & d_{7} & h_{5} & 0 & \kappa \lambda_{r}{ }^{*} \\
0 & 0 & 0 & 0 & 0 & 0 & (1-v) \delta & d_{8} & 0 & 0 \\
0 & 0 & 0 & 0 & 0 & 0 & v \delta & 0 & d_{9} & 0 \\
0 & 0 & 0 & \gamma(1-\eta) & q \rho_{s} & \phi & 0 & \rho_{r} & \varphi & d_{10}
\end{array}\right)
$$

Where, 


$$
\begin{gathered}
d_{1}=-\left(\sigma\left(\lambda_{s}{ }^{*}+\lambda_{r}{ }^{*}\right)+\theta+\mu\right), d_{2}=-\left(\lambda_{s}{ }^{*}+\lambda_{r}{ }^{*}+\mu\right), d_{3}=-\left(\lambda_{r}{ }^{*}+\alpha+\mu\right), d_{4}=-\left(\lambda_{r}{ }^{*}+\gamma+\mu\right), \\
d_{5}=-\left(\rho_{s}+\mu+d_{s}\right), d_{6}=-(\phi+\mu), d_{7}=-(\delta+\mu), d_{8}=-\left(\rho_{r}+\mu+d_{r}\right), d_{9}=-(\varphi+\mu), \\
d_{10}=-\left(\kappa\left(\lambda_{s}{ }^{*}+\lambda_{r}{ }^{*}\right)+\mu\right), e_{1}=-\sigma c \omega_{s} V^{*} / N^{*}, \\
e_{2}=-c \omega_{s} S^{*} / N^{*}, e_{3}=\frac{c \omega_{s}}{N^{*}}\left(S^{*}+\sigma V^{*}+\kappa R^{*}\right), \\
h_{1}=-\frac{\sigma c \omega_{r} V^{*}}{N^{*}}, h_{2}=-\frac{c \omega_{r} S^{*}}{N^{*}}, h_{3}=-\frac{c \omega_{r}}{N^{*}} H_{s}{ }^{*}, \\
h_{4}=-\frac{c \omega_{r}}{N^{*}} L_{s}^{*}, h_{5}=\frac{c \omega_{r}}{N^{*}}\left(S^{*}+\sigma V^{*}+H_{s}^{*}+L_{s}^{*}+\kappa R^{*}\right), h_{6}=\alpha \varepsilon(1-p), h_{7}=\alpha(1-\varepsilon)(1-p) \text { and } h_{8}=(1-q) \rho_{s} .
\end{gathered}
$$

The characteristic equation will be:

$$
\left|\begin{array}{cccccccccc}
d_{1}-\lambda & 0 & 0 & 0 & e_{1} & 0 & 0 & h_{1} & 0 & 0 \\
\theta & d_{2}-\lambda & 0 & 0 & e_{2} & 0 & 0 & h_{2} & 0 & 0 \\
\sigma \lambda_{s}{ }^{*} & \lambda_{s}{ }^{*} & d_{3}-\lambda & 0 & e_{3} & 0 & 0 & h_{3} & 0 & \kappa \lambda_{s}{ }^{*} \\
0 & 0 & \alpha \varepsilon(1-p) & d_{4}-\lambda & 0 & 0 & 0 & h_{4} & 0 & 0 \\
0 & 0 & \alpha(1-\varepsilon)(1-p) & \gamma \eta & d_{5}-\lambda & 0 & 0 & 0 & 0 & 0 \\
0 & 0 & \alpha p & 0 & 0 & d_{6}-\lambda & 0 & 0 & 0 & 0 \\
\sigma \lambda_{r}{ }^{*} & \lambda_{r}{ }^{*} & \lambda_{r}{ }^{*} & \lambda_{r}{ }^{*} & (1-q) \rho_{s} & 0 & d_{7}-\lambda & h_{5} & 0 & \kappa \lambda_{r}{ }^{*} \\
0 & 0 & 0 & 0 & 0 & 0 & (1-v) \delta & d_{8}-\lambda & 0 & 0 \\
0 & 0 & 0 & 0 & 0 & 0 & v \delta & 0 & d_{9}-\lambda & 0 \\
0 & 0 & 0 & \gamma(1-\eta) & q \rho_{s} & \phi & 0 & \rho_{r} & \varphi & d_{10}-\lambda
\end{array}\right|=0
$$

Now we apply the Gershgorin circle theorem [16] to determine the sign of the eigenvalues of the characteristic equation $U\left(E_{3}\right)-\lambda I \mid=0$. The matrix $J\left(E_{3}\right)$ is a strictly column diagonally dominant matrix. And also all diagonal elements of $J\left(E_{3}\right)$ are negative. Hence, all eigenvalues of $J\left(E_{3}\right)$ has negative real part if $R_{e f f}(\mathrm{DS})>1, R_{\text {eff }}(\mathrm{MDR})>1$ and $R^{*}<$ $\min \left\{\frac{\Lambda\left(\mu+d_{S}\right)}{c \omega_{s} \mu \kappa}, \frac{\Lambda\left(\mu+d_{r}\right)}{c \omega_{r} \mu \kappa}\right\}$. Therefore, the endemic equilibrium point where both TB strains co-exist $E_{3}$ is locally asymptotically stable if $R_{e f f}(\mathrm{DS})>1, R_{e f f}(\mathrm{MDR})>1$ and $R^{*}<\min \left\{\frac{\Lambda\left(\mu+d_{s}\right)}{c \omega_{s} \mu \kappa}, \frac{\Lambda\left(\mu+d_{r}\right)}{c \omega_{r} \mu \kappa}\right\}$.

\subsubsection{Global Stability of Endemic Equilibrium Point where Both TB Strains Co-exist, $E_{3}$}

Theorem-10:

The endemic equilibrium point where both TB strains co-exist $E_{3}$ of the system (9)-(18) is globally asymptotically stable if $R_{e f f}(\mathrm{DS})>1, R_{e f f}(\mathrm{MDR})>1$.

Proof: We use a graph-theoretic method as in [15] to construct a lyapunov function.

Define the functions:

$$
\begin{aligned}
& B_{1}=V-V^{*}-V^{*} \ln \frac{V}{V^{*}}, B_{2}=S-S^{*}-S^{*} \ln \frac{S}{S^{*}}, \\
& B_{3}=H_{s}-H_{s}{ }^{*}-H_{s}{ }^{*} \ln \frac{H_{s}}{H_{s}{ }^{*}}, B_{4}=L_{s}-L_{s}{ }^{*}-L_{s}{ }^{*} \ln \frac{L_{s}}{L_{s}{ }^{*}} \\
& B_{5}=I_{S}-I_{S}{ }^{*}-I_{S}{ }^{*} \ln \frac{I_{s}}{I_{S}{ }^{*}}, B_{6}=T_{S}-T_{S}{ }^{*}-T_{s}{ }^{*} \ln \frac{T_{S}}{T_{s}{ }^{*}}, \\
& B_{7}=E-E^{*}-E^{*} \ln \frac{E}{E^{*}}, \quad B_{8}=T_{r}-T_{r}{ }^{*}-T_{r}{ }^{*} \ln \frac{T_{r}}{T_{r}{ }^{*}} \\
& B_{9}=I_{r}-I_{r}{ }^{*}-I_{r}{ }^{*} \ln \frac{I_{r}}{I_{r}{ }^{*}}, B_{10}=R-R^{*}-R^{*} \ln \frac{R}{R^{*}}
\end{aligned}
$$

Where $E_{3}=\left(V^{*}, S^{*}, H_{s}{ }^{*}, L_{s}{ }^{*}, T_{s}{ }^{*}, I_{s}{ }^{*}, E^{*}, T_{r}{ }^{*}, I_{r}{ }^{*}, R^{*}\right)$ is the endemic equilibrium

Differentiating the function $B_{i}, i=1, \ldots, 10$ with respect to time we get:

$$
\begin{gathered}
\dot{B_{1}}=\left(1-\frac{V^{*}}{V}\right) \dot{V}, \dot{B_{2}}=\left(1-\frac{S^{*}}{S}\right) \dot{s}, \dot{B_{3}}=\left(1-\frac{H_{s}^{*}}{H_{s}}\right) \dot{H}_{s}, \dot{B_{4}}=\left(1-\frac{L_{s}^{*}}{L_{s}}\right) \dot{L_{s}}, \dot{B_{5}}=\left(1-\frac{I_{s}^{*}}{I_{s}}\right) \dot{I_{s}}, \dot{B_{6}}=\left(1-\frac{T_{s}^{*}}{T_{s}}\right) \dot{T_{s}}, \dot{B_{7}}= \\
\left(1-\frac{E^{*}}{E}\right) \dot{E}, \dot{B_{8}}=\left(1-\frac{I_{r}^{*}}{I_{r}}\right) \dot{I_{r}} \dot{B_{9}}=\left(1-\frac{T_{s}^{*}}{T_{s}}\right) \dot{T}_{s}, \text { and } \dot{B_{10}}=\left(1-\frac{R^{*}}{R}\right) \dot{R}
\end{gathered}
$$

Substituting their derivatives using the inequality $1-x+\ln x \leq 0$, for all $x>0$ and the endemic equilibrium point $E_{3}$ we have: 


$$
\begin{aligned}
& \dot{B_{1}} \leq \sigma V^{*} \lambda_{s}{ }^{*}\left(-\ln \frac{I_{s}}{I_{s}{ }^{*}}+\ln \frac{V^{*}}{V}-\frac{V^{*}}{V}+\frac{I_{s}}{I_{s}{ }^{*}}\right)+\sigma V^{*} \lambda_{r}{ }^{*}\left(\frac{I_{r}}{I_{r}{ }^{*}}-\ln \frac{V^{*}}{V}-\frac{I_{r} V}{I_{r}{ }^{*} V^{*}}\right)=: a_{15} G_{15}+a_{18} G_{18} . \\
& \dot{B_{2}} \leq \theta V^{*}\left(\frac{S^{*}}{S}-2-\ln \frac{V S^{*}}{V^{*} S}+\frac{V}{V^{*}}\right)+S^{*} \lambda_{s}{ }^{*}\left(-\ln \frac{I_{S}}{I_{S}{ }^{*}}+\ln \frac{S^{*}}{S}-\frac{S^{*}}{S}+\frac{I_{S}}{I_{s}{ }^{*}}\right)+S^{*} \lambda_{r}{ }^{*}\left(-\ln \frac{I_{r}}{I_{r}{ }^{*}}+\ln \frac{S^{*}}{S}-\frac{S^{*}}{S}+\frac{I_{r}}{I_{r}{ }^{*}}\right) \\
& =a_{21} G_{21}+a_{25} G_{25}+a_{28} G_{28}
\end{aligned}
$$

$\dot{B_{3}} \leq \lambda_{s}{ }^{*} S^{*}\left(\frac{I_{S} S}{I_{s}{ }^{*} S^{*}}-\frac{I_{S}}{I_{s}{ }^{*}}-\ln \frac{S}{S^{*}}\right)+\sigma V^{*} \lambda_{s}{ }^{*}\left(\frac{I_{S} V}{V^{*} I_{S}{ }^{*}}-\frac{I_{S}}{I_{S}{ }^{*}}-\ln \frac{V}{V^{*}}\right)+\kappa R^{*} \lambda_{s}{ }^{*}\left(\frac{I_{S} R}{R^{*} I_{s}{ }^{*}}-\frac{I_{S}}{I_{S}{ }^{*}}-\ln \frac{R}{R^{*}}\right)+\left(S^{*}+\sigma V^{*}+\kappa R^{*}\right) \lambda_{s}{ }^{*}\left(\frac{I_{S}}{I_{s}{ }^{*}}-\right.$ $\left.\frac{H_{S}}{H_{s}{ }^{*}}+\ln \frac{H_{S}}{H_{s}{ }^{*}}-\ln \frac{I_{s}}{I_{s}{ }^{*}}\right)+\lambda_{r}{ }^{*} H_{s}{ }^{*}\left(\frac{H_{S}}{H_{s}{ }^{*}}-1-\frac{H_{S}}{H_{s}{ }^{*}} \frac{\lambda_{r}{ }^{*}}{{ }^{*}}+\frac{\lambda_{r}}{\lambda_{r}{ }^{*}}\right)$.

$=: a_{32} G_{32}+a_{31} G_{31}+a_{310} G_{310}+a_{35} G_{35}+a_{38} G_{38}$

$\dot{B_{4}} \leq \alpha \varepsilon(1-p) H_{s}{ }^{*}\left(-\frac{L_{S}}{L_{S}{ }^{*}}+\ln \frac{L_{S}}{L_{s}{ }^{*}}-\ln \frac{H_{S}}{H_{s}{ }^{*}}+\frac{H_{S}}{H_{S}{ }^{*}}\right)+\lambda_{r}{ }^{*} L_{S}{ }^{*}\left(\frac{L_{S}}{L_{s}{ }^{*}}-2-\ln \frac{L_{s}}{L_{s}{ }^{*}} \frac{\lambda_{r}}{{ }_{r}{ }^{*}}+\frac{\lambda_{r}}{\lambda_{r}{ }^{*}}\right)=: a_{43} G_{43}+a_{48} G_{48}$.

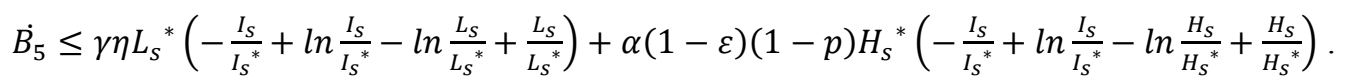

$$
=: a_{54} G_{54}+a_{53} G_{53}
$$

$$
\dot{B_{6}} \leq(\phi+\mu) T_{s}{ }^{*}\left(\frac{H_{s}}{H_{s}{ }^{*}}-\ln \frac{H_{s}}{H_{s}{ }^{*}}-\frac{T_{s}}{T_{s}{ }^{*}}+\ln \frac{T_{s}{ }^{*}}{T_{s}}\right)=: a_{63} G_{63}
$$

$\dot{B_{7}} \leq \lambda_{r}{ }^{*}\left(S^{*}+\sigma V^{*}+\kappa R^{*}+H_{s}{ }^{*}+L_{s}{ }^{*}\right)\left(\frac{I_{r}}{I_{r}{ }^{*}}-\frac{E}{E^{*}}-\ln \frac{I_{r}}{I_{r}{ }^{*}}+\ln \frac{E}{E^{*}}\right)+\lambda_{r}{ }^{*} S^{*}\left(\frac{I_{r} S}{I_{r}{ }^{*} S^{*}}-\frac{I_{r}}{I_{r}{ }^{*}}-\ln \frac{S}{S^{*}}\right)$

$$
\begin{gathered}
+\sigma \lambda_{r}{ }^{*} V^{*}\left(\frac{I_{r} V}{I_{r}{ }^{*} V^{*}}-\frac{I_{r}}{I_{r}{ }^{*}}-\ln \frac{V}{V^{*}}\right)+\kappa \lambda_{r}{ }^{*} R^{*}\left(\frac{I_{r} R}{I_{r}{ }^{*} R^{*}}-\frac{I_{r}}{I_{r}{ }^{*}}-\ln \frac{R}{R^{*}}\right)+\lambda_{r}{ }^{*} H_{s}{ }^{*}\left(\frac{I_{r} H_{s}}{I_{r}{ }^{*} H_{s}{ }^{*}}-\frac{I_{r}}{I_{r}{ }^{*}}-\ln \frac{H_{s}}{H_{s}{ }^{*}}\right) \\
+\lambda_{r}{ }^{*} L_{s}{ }^{*}\left(\frac{I_{r} L_{s}}{I_{r}{ }^{*} L_{s}{ }^{*}}-\frac{I_{r}}{I_{r}{ }^{*}}-\ln \frac{L_{s}}{L_{s}{ }^{*}}\right)+(1-q) \rho_{s} I_{s}{ }^{*}\left(\frac{I_{s}}{I_{s}{ }^{*}}-\frac{E}{E^{*}}-\ln \frac{I_{s}}{I_{s}{ }^{*}}+\ln \frac{E}{E^{*}}\right) \\
=: a_{78} G_{78}+a_{72} G_{72}+a_{71} G_{71}+a_{73} G_{73}+a_{74} G_{74}+a_{710} G_{710}+a_{75} G_{75} \\
\dot{B_{8}} \leq(1-v) \delta E^{*}\left(\frac{E}{E^{*}}-\frac{I_{r}}{I_{r}{ }^{*}}-\ln \frac{E}{E^{*}}+\ln \frac{I_{r}}{I_{r}{ }^{*}}\right)=: a_{87} G_{87} \\
\dot{B}_{9} \leq v \delta E^{*}\left(\frac{E}{E^{*}}-\frac{T_{r}}{T_{r}{ }^{*}}-\ln \frac{E}{E^{*}}+\ln \frac{T_{r}}{T_{r}{ }^{*}}\right)=: a_{97} G_{97} .
\end{gathered}
$$

$B_{10} \leq \rho_{s} I_{s}{ }^{*}\left(\frac{I_{s}}{I_{s}{ }^{*}}-\ln \frac{I_{s}}{I_{s}{ }^{*}}+\ln \frac{R}{R^{*}}-\frac{R}{R^{*}}\right)+\gamma(1-\eta) L_{s}{ }^{*}\left(-\frac{R}{R^{*}}+\ln \frac{R}{R^{*}}-\ln \frac{L_{s}}{L_{s}{ }^{*}}+\frac{L_{s}}{L_{s}{ }^{*}}\right)+\phi T_{s}{ }^{*}\left(-\frac{R}{R^{*}}+\ln \frac{R}{R^{*}}-\ln \frac{T_{s}}{T_{s}{ }^{*}}+\frac{T_{s}}{T_{s}{ }^{*}}\right)$

$$
\begin{gathered}
+\kappa \lambda_{s}{ }^{*} R^{*}\left(\frac{R}{R^{*}}-2-\ln \frac{R}{R^{*}} \frac{I_{s}}{I_{s}{ }^{*}}+\frac{I_{s}}{I_{s}{ }^{*}}\right)+\rho_{r} I_{r}{ }^{*}\left(\frac{I_{r}}{I_{r}{ }^{*}}-\ln \frac{I_{r}}{I_{r}{ }^{*}}+\ln \frac{R}{R^{*}}-\frac{R}{R^{*}}\right)+\varphi T_{r}{ }^{*}\left(\frac{T_{r}}{T_{r}{ }^{*}}-\ln \frac{T_{r}}{T_{r}{ }^{*}}+\ln \frac{R}{R^{*}}-\frac{R}{R^{*}}\right) \\
+\kappa \lambda_{r}{ }^{*} R^{*}\left(-\ln \frac{R}{R^{*}}-\ln \frac{I_{r}}{I_{r}{ }^{*}}-2+\frac{R}{R^{*}}+\frac{I_{r}}{I_{r}{ }^{*}}\right) \\
=: a_{105 a} G_{105 a}+a_{104} G_{104}+a_{106} G_{106}+a_{105 b} G_{105 b}+a_{108 a} G_{108 a}+a_{109} G_{109}+a_{108 b} G_{108 b} .
\end{gathered}
$$

Where,

$$
\begin{gathered}
a_{15}=a_{31}=\sigma V^{*} \lambda_{s}{ }^{*}, a_{18}=a_{71}=\sigma V^{*} \lambda_{r}{ }^{*}, \\
a_{21}=\theta V^{*}, a_{25}=a_{32}=S^{*} \lambda_{s}{ }^{*}, a_{28}=a_{72}=S^{*} \lambda_{r}{ }^{*}, \\
a_{310}=\kappa R^{*} \lambda_{s}{ }^{*}, a_{35}=\left(S^{*}+\sigma V^{*}+\kappa R^{*}\right) \lambda_{s}{ }^{*}, \\
a_{38}=a_{73}=\lambda_{r}{ }^{*} H_{s}{ }^{*}, a_{43}=\alpha \varepsilon(1-p) H_{s}{ }^{*}, a_{48}=a_{74}=\lambda_{r}{ }^{*} L_{s}{ }^{*}, \\
a_{54}=\gamma \eta L_{s}{ }^{*}, a_{53}=\alpha(1-\varepsilon)(1-p) H_{s}{ }^{*}, \\
a_{63}=(\phi+\mu) T_{s}{ }^{*}, a_{710}=\kappa \lambda_{r}{ }^{*} R^{*}, a_{75}=(1-q) \rho_{s} I_{s}{ }^{*}, a_{87}=(1-v) \delta E^{*}, a_{97}=v \delta E^{*}, a_{105 a}=\rho_{s} I_{s}{ }^{*}, \\
a_{104}=\gamma(1-\eta) L_{s}{ }^{*}, a_{106}=\phi T_{s}{ }^{*}, a_{105 b}=\kappa \lambda_{s}{ }^{*} R^{*},
\end{gathered}
$$$$
a_{108 a}=\rho_{r} I_{r}{ }^{*}, a_{109}=\varphi T_{r}{ }^{*}, a_{108 b}=\kappa \lambda_{r}{ }^{*} R^{*} \text { and all other } a_{i j}=0 .
$$ 
Along each cycle of the associated weighted digraph $\mathrm{G}(\mathrm{B})$ :

$$
\begin{aligned}
& G_{35}+G_{53}=\left(\frac{I_{S}}{I_{S}{ }^{*}}-\frac{H_{S}}{H_{S}{ }^{*}}+\ln \frac{H_{S}}{H_{S}{ }^{*}}-\ln \frac{I_{S}}{I_{S}{ }^{*}}\right)+\left(-\frac{I_{S}}{I_{S}{ }^{*}}+\ln \frac{I_{S}}{I_{S}{ }^{*}}-\frac{H_{S}}{H_{S}{ }^{*}}+\frac{H_{S}}{H_{S}{ }^{*}}\right)=0, \\
& G_{35}+G_{43}+G_{54}=\left(\frac{I_{S}}{I_{S}{ }^{*}}-\frac{H_{S}}{H_{S}{ }^{*}}+\ln \frac{H_{S}}{H_{S}{ }^{*}}-\ln \frac{I_{S}}{I_{S}{ }^{*}}\right)+\left(-\frac{L_{S}}{L_{S}{ }^{*}}+\ln \frac{L_{S}}{L_{S}{ }^{*}}-\ln \frac{H_{S}}{H_{S}{ }^{*}}+\frac{H_{S}}{H_{S}{ }^{*}}\right)+\left(-\frac{I_{S}}{I_{S}{ }^{*}}+\ln \frac{I_{S}}{I_{S}{ }^{*}}-\ln \frac{L_{S}}{L_{S}{ }^{*}}+\frac{L_{S}}{L_{S}{ }^{*}}\right)=0 \text {, } \\
& G_{34}+G_{43}=\left(\frac{I_{r}}{I_{r}{ }^{*}}-\frac{E}{E^{*}}-\ln \frac{I_{r}}{I_{r}{ }^{*}}+\ln \frac{E}{E^{*}}\right)+\left(\frac{E}{E^{*}}-\frac{I_{r}}{I_{r}{ }^{*}}-\ln \frac{E}{E^{*}}+\ln \frac{I_{r}}{I_{r}{ }^{*}}\right)=0 .
\end{aligned}
$$

By Proposition 1.3 of [15], there exists $c_{i}>0, i=1, \ldots, 10$ such that $B=\sum_{i=1}^{10} c_{i} B_{i}$ is a Lyapunov function for equations (9)-(18). The relations between $c_{i}$ 's can be derived from Theorems 3.3 and 3.4 of [15] such that:

$$
a_{97}>0, d^{+}(7)=1 \text { implies } c_{9}=c_{7} . a_{106}>0, d^{+}(6)=1 \text { implies } c_{10}=c_{6} \frac{\left(a_{105 a}+a_{104}+a_{106}+a_{105 b}+a_{108 a}+a_{109}+a_{108 b}\right)}{a_{106}} .
$$

$$
a_{87}>0, d^{-}(8)=1 \text { implies } c_{8}=\frac{c_{1} a_{18}+c_{2} a_{28}+c_{3} a_{38}+c_{4} a_{48}+c_{7} a_{78}+c_{10}\left(a_{108 a}+a_{108 b}\right)}{a_{87}}
$$

Therefore, $\quad B=c_{1} B_{1}+c_{2} B_{2}+c_{3} B_{3}+c_{4} B_{4}+c_{5} B_{5}+$ $c_{6} B_{6}++c_{7} B_{7}+c_{10} B_{10}+c_{9} B_{9}++c_{10} B_{10}$ is a Lyapunov function for (9)- (18). Therefore, $E_{3}$ is globally asymptotically stable in the interior of $\Omega$ when $R_{\text {eff }}(\mathrm{DS})>1$ and $R_{e f f}(\mathrm{MDR})>1$.

\section{Numerical Simulations}

We perform some numerical experimentation on the tuberculosis model (9)-(18). This is done by using a set of parameter values whose sources are mainly from Federal Democratic Republic of Ethiopia Ministry of Health (EMH), Ethiopia Demographics Profile (EDP), world health organization (WHO) reports and other related literatures as well as estimation in order to have more realistic simulation results. We take initial condition from the data of Ministry of Health of Ethiopia [17]: $V(0)=9436405, I_{s}(0)=42139, T_{s}(0)=83546, E(0)=$ $8098, I_{r}(0)=774, T_{r}(0)=468$ and $\mathrm{R}(0)=3597$. We assumed that more than half of the population (62\%) belongs to susceptible class $S(0)=62355690$ and that a big percentage about $33 \%$ is infected with TB in latent stage that is $H_{S}(0)=3,000000$, and $L_{s}(0)=30000000$, . This is justified from the fact that "about one third of the world's

\begin{tabular}{|c|c|c|c|}
\hline Descriptions & Symbols & Value & Data Source \\
\hline Recruitment of the population & $\Lambda$ & 3845257 & {$[18]$} \\
\hline Proportions new born vaccinated & $\psi$ & 0.49 & {$[18],[17]$} \\
\hline Natural death rate & $\mu$ & 0.0077 & {$[18]$} \\
\hline the rate of inefficacy of vaccine individuals & $\sigma$ & 0.2 & [19] \\
\hline the rate of vaccine waning & $\theta$ & 0.0667 & [19] \\
\hline Probability of acquiring TB infections per contact with one active drug sensitive TB & $\omega_{s}$ & 0.2 & [20] \\
\hline Probability of acquiring TB infections per contact with one active MDR TB & $\omega_{r}$ & 0.3 & {$[21]$} \\
\hline The rate of progression of individuals from early latently infected with drug sensitive TB. & $\alpha$ & 0.5 & [19] \\
\hline Death rate due to the drug sensitive strain disease & $d_{s}$ & 0.00025 & {$[22,23]$} \\
\hline Death rate due to the MDR strain disease & $d_{r}$ & 0.105 & {$[23,24]$} \\
\hline Proportion of $H_{s}$ move to $T_{s}$ & $p$ & 0.2 & [17] \\
\hline The portion of $L_{s}$ enter in to $I_{s}$ & $\eta$ & 0.5 & [19] \\
\hline Progression rate from $L_{s}$ & $\gamma$ & 0.1 & [19] \\
\hline Progression rate from latency multi-drug resistant tuberculosis. & $\delta$ & 0.55 & [17] \\
\hline The recovery rate infectious individuals MDR strain. & $\rho_{r}$ & 0.498 & {$[23,24]$} \\
\hline Proportion of infectious individuals with DSTB who enters to R. & $q$ & 0.18 & [17] \\
\hline The portion of $E$ who screened for treatment & $v$ & 0.065 & [17] \\
\hline Rate of individuals move from $T_{s}$ to $\mathrm{R}$ & $\phi$ & 0.94 & [17] \\
\hline Rate of individuals move from $T_{r}$ to $\mathrm{R}$ & $\varphi$ & 0.88 & [17] \\
\hline Acquired immunity due to previous treatment. & $\kappa$ & 0.06 & [25] \\
\hline
\end{tabular}
population has latent TB", as it is indicated from the webpage of the World Health Organization (WHO, 2017).

\subsection{Estimation of Parameters}

Table 2. Model parameter estimation.

\subsection{Numerical Simulation for the Reproduction Number}

We discussed on the relation between effective reproduction number and the parameters involved in it. Now we consider the parameters that involve in both $R_{e f f}(\mathrm{DS})$ and $R_{e f f}(\mathrm{MDR})$ and discuss on their impact on the transmission of DS-TB and/or MDR-TB strains. Here five 
parameters are involve in common for both effective reproduction numbers $R_{e f f}(\mathrm{DS})$ and $R_{\text {eff }}(\mathrm{MDR})$.

Let us consider the parameter, the number of effective contacts $\mathrm{c}$ as a variable and keeping all other parameters constant and written the reproduction numbers as a function of $\mathrm{c}, R_{\text {eff }}(\mathrm{DS})(c)=0.0934 c$ and $R_{\text {eff }}(\mathrm{MDR})(c)=$ $0.4348 c$. Consider the rate of inefficacy of vaccine individuals $\sigma$ as a variable and keeping all other parameters as constant, the reproduction numbers can be written as a function of $\sigma: R_{\text {eff }}(\mathrm{DS})(\sigma)=0.0547 \sigma+1.0164$ and $R_{\text {eff }}(\mathrm{MDR})(\sigma)=0.2549 \sigma+4.7355$

In the figure 3 the lines $R_{\text {eff }}(\mathrm{DS})(c)=0.0934 c$ and $R_{\text {eff }}(\mathrm{MDR})(c)=0.4348 c$ intersect with $R_{\text {eff }}(\mathrm{MDR})=$ $R_{\text {eff }}(\mathrm{DS})=1$ intersect at the values of $c=10.7$ and $c=2.3$ respectively. Thus, $R_{\text {eff }}(D S)<1$ when the contact rate, $c<10.7$ and $R_{\text {eff }}$ (DS) $>1$ when $c>10.7$. For the value of $2.3<c<10.7$ only MDR-TB spread in the society. Whereas $R_{\text {eff }}(\mathrm{MDR})<1$ when the number of effective

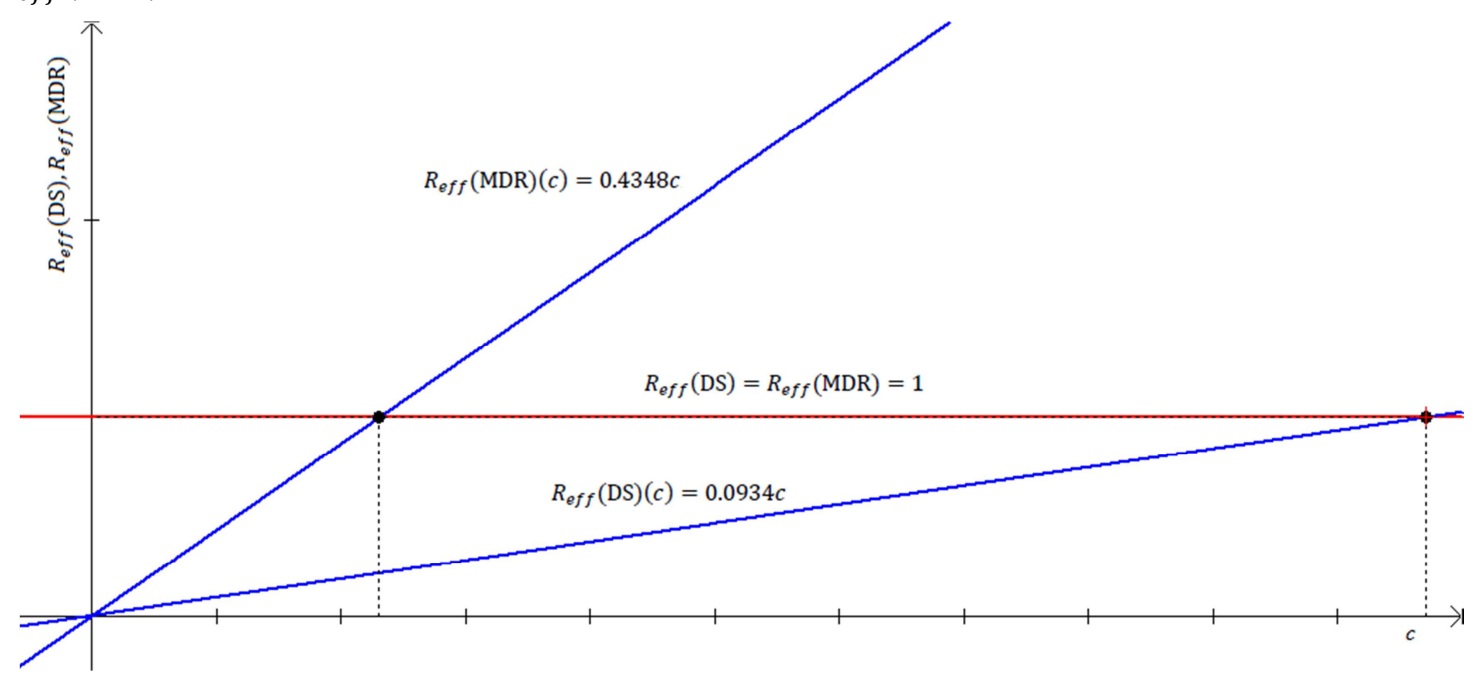

Figure 3. Graph of the reproduction number versus the number of effective contacts $c$.

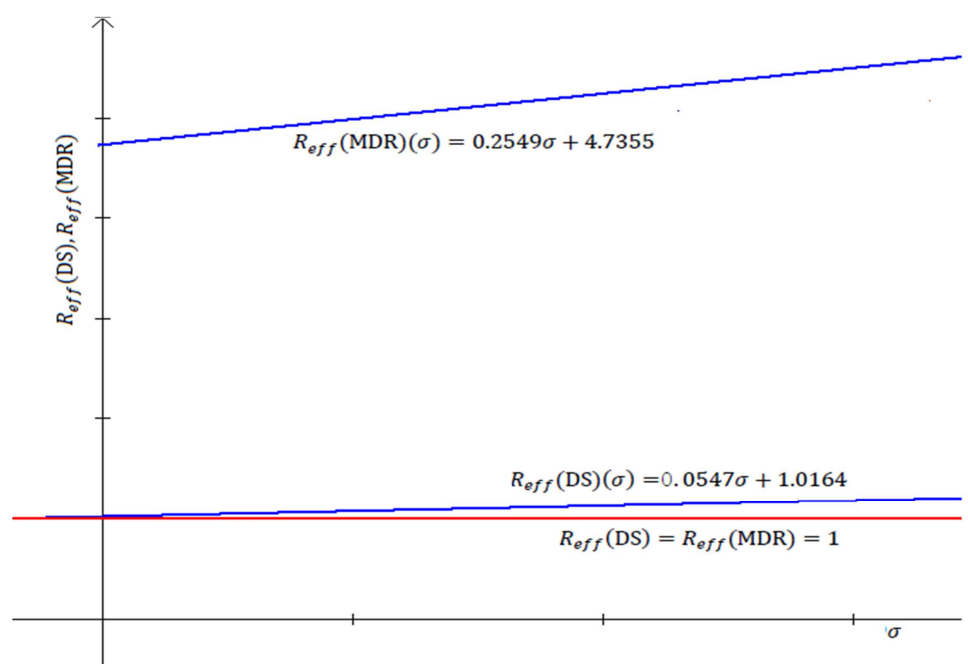

Figure 4. The reproduction number versus the rate of inefficacy of vaccine. contacts, $c<2.3$ and $R_{\text {eff }}(\mathrm{MDR})>1$ when $c>2.3$. This implies the TB disease spreads in the community when $c>2.3$ and eliminate if $c<2.3$. Figure 4 shows that both $R_{e f f}(\mathrm{DS})(\sigma)$ and $R_{\text {eff }}(\mathrm{MDR})(\sigma)$ are above $R_{\text {eff }}(\mathrm{DS})=$ $R_{\text {eff }}(\mathrm{MDR})=1$, thus for every values of $\sigma$ the both strains of the TB disease spread in the society. Of course the transmission of MDR-TB is higher than DS-TB.

Consider the rate of vaccine waning $\theta$ as a variable and keeping all other parameters as constant, the reproduction number can be written as a function of $\theta: R_{\text {eff }}(\mathrm{DS})(\theta)=$ $\frac{0.005+1.0712 \theta}{\theta+0.0077}$ and $R_{\text {eff }}(\mathrm{MDR})(\theta)=\frac{4.9824(\theta+0.0047)}{\theta+0.0077}$. Consider the proportions new born vaccinated $\psi$ as a variable and keeping all other parameters as constant, the reproduction number can be written as a function of $\psi: R_{\text {eff }}(\mathrm{DS})(\psi)=$ $1.0744-0.0895 \psi \quad$ and $\quad R_{\text {eff }}(\mathrm{MDR})(\psi)=4.9904-$ $0.4159 \psi$. 


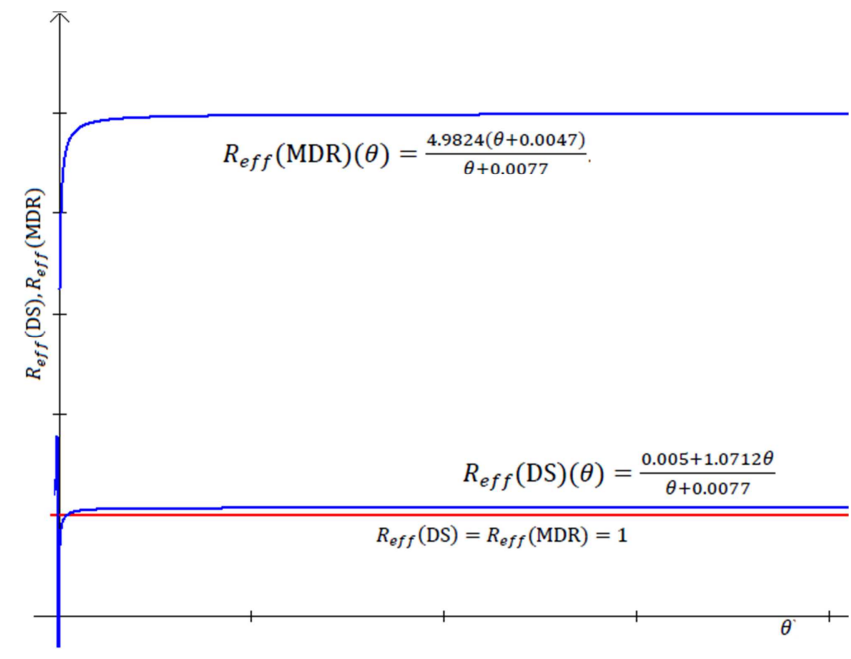

Figure 5. Graph of the reproduction numbers $R_{e f f}(D S)$ and $R_{\text {eff }}(M D R)$ versus the rate of vaccine waning $\theta$.

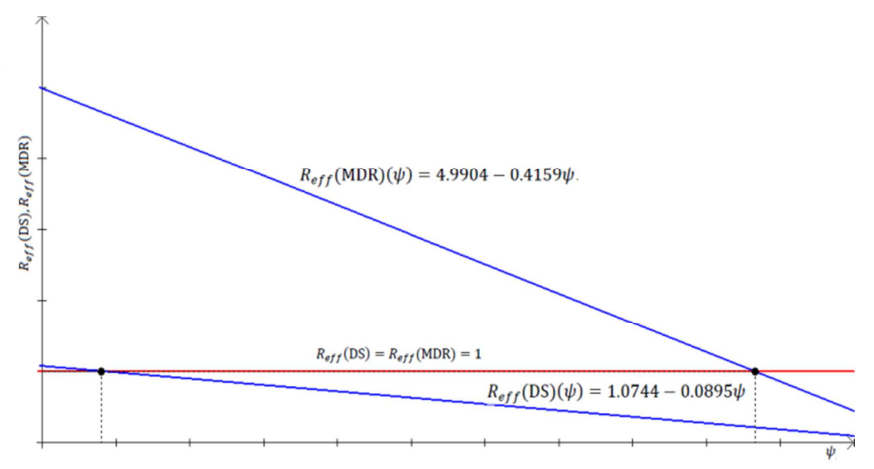

Figure 6. Graph of the reproduction numbers $R_{\text {eff }}(D S)$ and $R_{\text {eff }}(M D R)$ versus Proportions new born vaccinated $\psi$.

In figure 5 the curve $R_{\text {eff }}(\mathrm{DS})=\frac{0.005+1.0712 \theta}{\theta+0.0077}$ and the line $R_{\text {eff }}(\mathrm{DS})=1$ intersect at $\theta=0.038$, then $R_{\text {eff }}(\mathrm{DS})<1$ when $\theta<0.038$ and $R_{\text {eff }}$ (DS) $>1$ when $\theta>0.038$. But the curve $R_{e f f}(M D R)(\theta)=\frac{4.9824(\theta+0.0047)}{\theta+0.0077}$ is above $R_{\text {eff }}(\mathrm{MDR})=1$. This implies the MDR-TB spreads in the community for every value of $\theta$. And for the value of $\theta<0.038$ the DS-TB does not spread in the society. In figure 6 the curve $R_{\text {eff }}(\mathrm{DS})(\psi)$ and the line $R_{\text {eff }}(\mathrm{DS})=1$ intersect at $\psi=0.8$, then $R_{e f f}$ (DS) $<1$ when $\psi>0.8$ and $R_{e f f}(\mathrm{DS})>1$ when $\psi<0.8$. And $R_{\text {eff }}(\mathrm{MDR})>1$ for all values of $\psi$. This shows that the MDR-TB exist always, but DS-TB spreads for $\psi<0.8$.

Consider the Proportion of latently infected drug sensitive TB at early stage for treatment $p$ as a variable and keeping all other parameters as constant, the reproduction number can be written as a function of $p: R_{\text {eff }}(\mathrm{DS})(p)=1.2941(1-p)$. Again, consider the recovery rate infectious individuals DSTB strain disease $\rho_{s}$ as a variable and keeping all other parameters as constant, then the reproduction number can be written as a function of $\rho_{s}: R_{e f f}(\mathrm{DS})\left(\rho_{s}\right)=\frac{0.8537}{\rho_{S}+0.00795}$

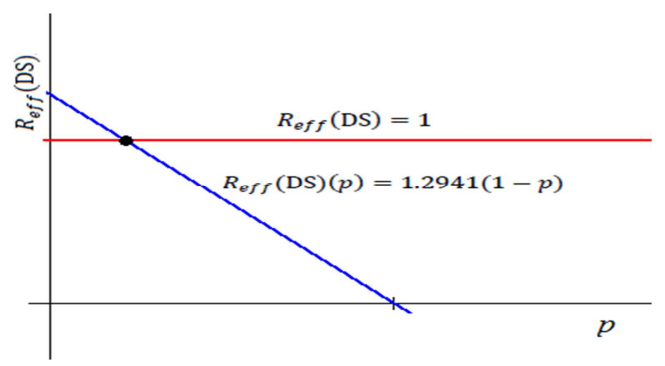

Figure 7. Graph of the reproduction number $R_{\text {eff }}(D S)$ versus the Proportion of latently infected DS TB at early stage for treatment $p$.

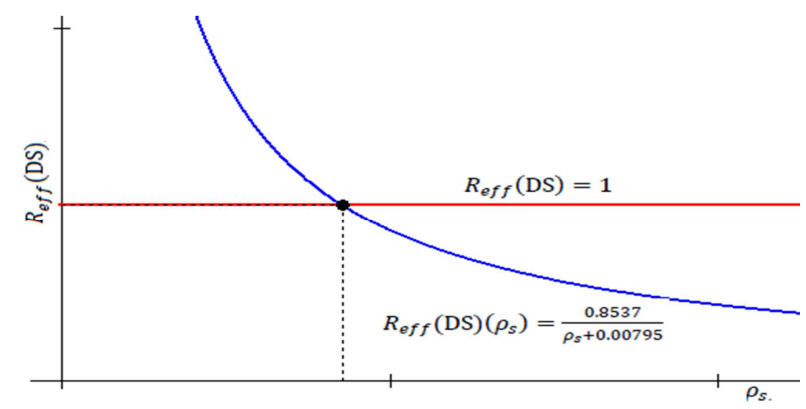

Figure 8. Graph of the reproduction number $R_{\text {eff }}(D S)$ versus the parameter $\rho_{S}$.

In figure 7 the curve $R_{\text {eff }}(\mathrm{DS})(p)=1.2941(1-p)$ and the line $R_{\text {eff }}(\mathrm{DS})=1$ intersect at $p=0.22, R_{\text {eff }}(\mathrm{DS})<1$ when $p>0.22$ and $R_{\text {eff }}$ (DS) $>1$ when $p<0.22$. In figure 8 the curve $R_{\text {eff }}(\mathrm{DS})\left(\rho_{s}\right)=\frac{0.8537}{\rho_{s}+0.00795}$ and the line $R_{e f f}(\mathrm{DS})=1$ intersect at $\rho_{s}=0.85, R_{e f f}(\mathrm{DS})<1$ when $\rho_{s}>0.85$ and $R_{\text {eff }}$ (DS) $>1$ when $\rho_{s}<0.85$.

The reproduction number $R_{\text {eff }}(\mathrm{MDR})$ can also be given as a function of the recovery rate infectious MDRTB individuals $\rho_{r}$ : keeping all other parameters as constant, $R_{e f f}(\mathrm{MDR})\left(\rho_{r}\right)=\frac{2.9181}{\rho_{r}+0.1127}$. And the reproduction number $R_{e f f}(\mathrm{MDR})$ can also be given as a function of the portion of $E$ enter in to $I_{r}, v$ : keeping all other parameters as constant, $R_{e f f}(\mathrm{MDR})(v)=5.1225(1-v)$.

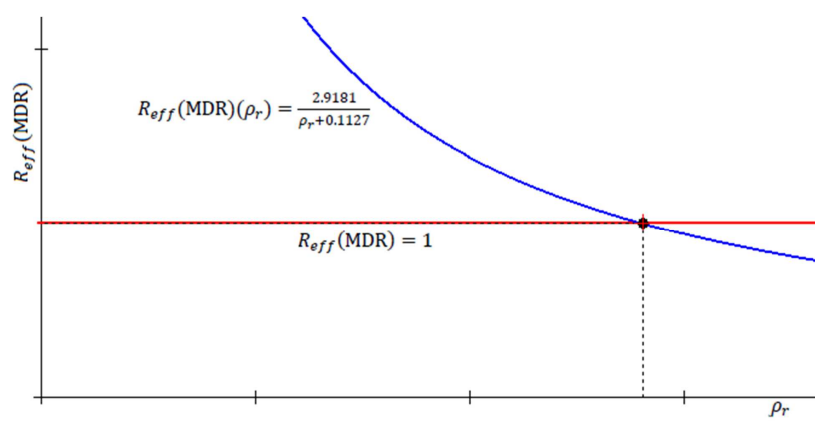

Figure 9. Graph of the reproduction number $R_{\text {eff }}(M D R)$ versus the parameter $\rho_{r}$. 


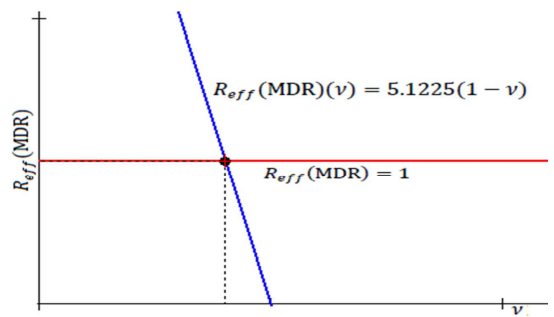

Figure 10. Graph of the reproduction number $R_{\text {eff }}(M D R)$ versus the parameter $v$.

In figure 9 the curve $R_{e f f}(\mathrm{MDR})\left(\rho_{r}\right)=\frac{2.9181}{\rho_{r}+0.1127}$ and the line $R_{e f f}(\mathrm{MDR})=1$ intersect at $\rho_{r}=2.8, R_{e f f}(\mathrm{MDR})<1$

$$
\begin{aligned}
& R_{e f f}=\max \left\{R_{e f f}(\mathrm{DS}), R_{e f f}(\mathrm{MDR})\right\}=R_{e f f}(\mathrm{DS}) \\
= & c \omega_{s}\left(\frac{(\theta+(1-\psi) \mu)+\mu \sigma \psi}{(\mu+\theta)}\right) \frac{(\alpha \varepsilon(1-p) \gamma \eta+\alpha(\gamma+\mu)(1-\varepsilon)(1-p))}{(\alpha+\mu) \times(\gamma+\mu) \times\left(\rho_{s}+\mu+d_{S}\right)} .
\end{aligned}
$$

Therefore, we evaluate the nonzero sensitivity indices of $R_{e f f}(D S)$ with respect to the parameters as follows:

$$
\begin{gathered}
\Pi_{c}^{R_{e f f}(\mathrm{DS})}=\Pi_{\omega_{s}}^{R_{e f f}(\mathrm{DS})}=1, \Pi_{\psi}^{R_{e f f}(\mathrm{DS})}=\frac{\mu \psi(\sigma-1)}{\theta+\mu+\mu \psi(\sigma-1)}<0, \Pi_{\sigma}^{R_{e f f}(\mathrm{DS})}=\frac{\psi \sigma \mu}{\theta+\mu+\psi \mu(\sigma-1)}>0, \Pi_{d_{s}}^{R_{e f f}(\mathrm{DS})}=-\frac{d_{s}}{\left(\rho_{s}+\mu+d_{s}\right)}<0, \\
\Pi_{\theta}^{R_{e f f}(\mathrm{DS})}=\frac{\psi \mu \theta(1-\sigma)}{(\mu+\theta)[\theta+\mu+\mu \psi(\sigma-1)]}>0, \Pi_{\alpha}^{R_{e f f}(\mathrm{DS})}=\frac{\mu}{\alpha+\mu}>0,
\end{gathered}
$$

$\Pi_{p}^{R_{e f f}(\mathrm{DS})}=-\frac{p}{1-p}<0, \Pi_{\rho_{S}}^{R_{e f f}(\mathrm{DS})}=-\frac{\rho_{S}}{\rho_{S}+\mu+d_{S}}<0, \quad \Pi_{\gamma}^{R_{e f f}(\mathrm{DS})}=\left\{\frac{(\alpha \varepsilon(1-p) \eta+\alpha(1-\varepsilon)(1-p)) \gamma}{(\alpha \varepsilon(1-p) \gamma \eta+\alpha(\gamma+\mu)(1-\varepsilon)(1-p))}-\frac{\gamma}{(\gamma+\mu)}\right\}>0, \Pi_{\varepsilon}^{R_{e f f}(\mathrm{DS})}=$ $\frac{\varepsilon(1-p)(\gamma \eta-(\gamma+\mu))}{(\varepsilon(1-p) \gamma \eta+(\gamma+\mu)(1-\varepsilon)(1-p))}<0$,

$\Pi_{\eta}^{R_{e f f}(\mathrm{DS})}=\frac{\eta \varepsilon \gamma}{(\varepsilon \gamma \eta+(\gamma+\mu)(1-\varepsilon))}>0$

If $R_{e f f}(\mathrm{MDR})>R_{e f f}(\mathrm{DS})$, then we have

$$
R_{e f f}=\max \left\{R_{e f f}(\mathrm{DS}), R_{e f f}(\mathrm{MDR})\right\}=R_{e f f}(\mathrm{MDR})=\left(\frac{\sigma \psi \mu+(\theta+(1-\psi) \mu)}{(\mu+\theta)}\right) \frac{c \omega_{r}(1-v) \delta}{(\delta+\mu) \times\left(\rho_{r}+\mu+d_{r}\right)} .
$$

Therefore, we evaluated the nonzero sensitivity indices of $R_{e f f}(M D R)$ with respect to the parameters as follows:

$$
\begin{gathered}
\Pi_{c}^{R_{e f f}(\mathrm{MDR})}=\Pi_{\omega_{r}}^{R_{e f f}(\mathrm{MDR})}=1, \Pi_{\psi}^{R_{e f f}(\mathrm{MDR})}=\frac{\mu \psi(\sigma-1)}{(\theta+(1-\psi) \mu)+\mu \sigma \psi}<0 \\
\Pi_{\sigma}^{R_{e f f}(\mathrm{MDR})}=\frac{\mu \sigma \psi}{[\theta+(1-\psi) \mu)+\sigma \psi]}>, \Pi_{v}^{R_{e f f}(\mathrm{MDR})}=-\frac{v}{1-v}<0, \\
\Pi_{\theta}^{R_{e f f}(\mathrm{MDR})}=\frac{\theta \psi \mu(1-\sigma)}{(\mu+\theta)[\theta+(1-\psi) \mu+\mu \sigma \psi]}>0, \Pi_{\delta}^{R_{e f f}(\mathrm{MDR})}=\frac{\mu}{(\delta+\mu)}>0, \\
\Pi_{\rho_{r}}^{R_{e f f}(\mathrm{MDR})}=-\frac{\rho_{r}}{\left(\rho_{r}+\mu+d_{r}\right)}<0, \Pi_{d_{r}}^{R_{e f f}(\mathrm{MDR})}=-\frac{d_{r}}{\left(\rho_{r}+\mu+d_{r}\right)}<0,
\end{gathered}
$$

Using the data in table 2 the resulting sensitivity indices of $R_{\text {eff }}(\mathrm{DS})$ and $R_{\text {eff }}(\mathrm{MDR})$ to the different parameters which involve in the reproduction numbers $R_{e f f}(\mathrm{DS})$ and $R_{\text {eff }}$ (MDR) respectively are shown in table 3 with the order from most sensitive to the least:

Table 3. Sensitivity indices of $R_{\text {eff }}(M D R)$ and $R_{\text {eff }}(M D R)$ with respect to each parameter involved in $R_{\text {eff }}(M D R)$ and $R_{\text {eff }}(M D R)$ respectively.

\begin{tabular}{llll}
\hline Parameters & $\begin{array}{l}\text { Sensitivity index } \\
\text { of } \boldsymbol{R}_{\text {eff }} \text { (DS) }\end{array}$ & Parameters & $\begin{array}{l}\text { Sensitivity index } \\
\text { of } \boldsymbol{R}_{\text {eff }}(\text { MDR })\end{array}$ \\
\hline$c$ & 1 & $c$ & 1 \\
$\omega_{s}$ & 1 & $\omega_{r}$ & 1 \\
\hline
\end{tabular}

\begin{tabular}{llll}
\hline Parameters & $\begin{array}{l}\text { Sensitivity index } \\
\text { of } \boldsymbol{R}_{\text {eff }}(\mathbf{D S})\end{array}$ & Parameters & $\begin{array}{l}\text { Sensitivity index } \\
\text { of } \boldsymbol{R}_{\text {eff }}(\mathbf{M D R})\end{array}$ \\
\hline$\rho_{s}$ & -0.99 & $\rho_{r}$ & -0.82 \\
$\varepsilon$ & -0.93 & $d_{r}$ & -0.17 \\
$\eta$ & 0.81 & $v$ & -0.07 \\
$p$ & -0.25 & $\psi$ & -0.042 \\
$\psi$ & -0.11 & $\theta$ & 0.038 \\
$\gamma$ & 0.058 & $\delta$ & 0.014 \\
$\theta$ & 0.038 & $\sigma$ & 0.011 \\
$\alpha$ & 0.015 & & \\
$\sigma$ & 0.011 & & \\
$d_{s}$ & -0.0003 & & \\
\hline
\end{tabular}




\section{Discussion}

In this work we considered non-linear dynamical system to study the dynamics of a two strain Tuberculosis disease. The effective reproduction number is: $R_{\text {eff }}=c\left(\frac{\sigma \psi \mu+(\theta+(1-\psi) \mu)}{\theta+\mu}\right) \times$ $\max \left\{\frac{\omega_{s}(\alpha \varepsilon(1-p) \gamma \eta+\alpha(\gamma+\mu)(1-\varepsilon)(1-p))}{(\alpha+\mu)(\gamma+\mu)\left(\rho_{s}+\mu+d_{s}\right)}, \frac{\omega_{r}(1-v) \delta}{(\delta+\mu)\left(\rho_{r}+\mu+d_{r}\right)}\right\}$. Then the numerical value of $R_{\text {eff }}=\max \{1.03,4.78\}=4.78$. In the figure $3, R_{e f f}$ (DS) $<1$ when the number of contact of susceptible individuals with an infectious, $c<10.7$ and $R_{\text {eff }}(\mathrm{DS})>1$ when $c>10.7$. Moreover, $R_{\text {eff }}(\mathrm{MDR})<1$ when number of contact of susceptible individuals with an infectious, $c<2.3$ and $R_{\text {eff }}(\mathrm{MDR})>1$ when $c>2.3$. Thus the only MDR TB spreads in the society when the value of $2.3<c<10$; both strains spread in the society if $c>10.7$ and both strains do not spread in the society if $c<2.3$. Figure 4 shows that both $R_{e f f}(\mathrm{DS})>1$ and $R_{e f f}(\mathrm{MDR})>1$ for every values of $\sigma$, therefore both strains of the TB disease spread in the society what ever the value of $\sigma$ is. Ofcourse the transmision of MDR-TB is higher than DS-TB.

In figure 5 shows that $R_{\text {eff }}(\mathrm{DS})<1$ when $\theta<0.038$ and $R_{e f f}(\mathrm{DS})>1$ when $\theta>0.038$. But $R_{\text {eff }}(\mathrm{MDR})>1$ for all values of $\theta$. This implies the MDR-TB spreads in the community for every value of $\theta$. And for the value of $\theta<0.038$ the DS-TB does not spread in the society. That is, if $\theta<0.038$ the only MDRTB spreads in the community and boths DSTB and MDRTB spreads in the community when $\theta>0.038$. From figure 6 we observe that $R_{\text {eff }}(\mathrm{DS})<1$ when $\psi>0.8$ and $R_{e f f}$ (DS) $>1$ when $\psi<0.8$ while, $R_{e f f}(\mathrm{MDR})>1$ for all values of $\psi$. This shows that the MDR-TB spreads in the society for all values of $\psi$, but DSTB spreads for $\psi<0.8$. Implies both strain spreads in the community if $\psi<0.8$ and only MDRTB spreads in the community if $\psi>0.8$. This indicates that giving $\mathrm{BCG}$ vaccine has no significant impact in the control of MDRTB; however we can reduce DSTB by BCG vaccine.

From figures (3-10) and the sensitivity index of effective reproduction number (Table 3) we observe that the parameters contact rate $\mathrm{c}$, the rate of inefficacy of vaccine individuals $\sigma$, the rate of vaccine waning $\theta$, the probability of transmission $\omega_{s}$ and $\omega_{r}$, the rate of progression of individuals from early latently infected with drug sensitive TB $\alpha$, the progression rate from Long latently infected DSTB strain $\gamma$, the portion of $L_{s}$ enter in to $I_{s}, \eta$ and the progression rate from latency MDR-TB $\delta$ have positive contribution in the transmission of TB disease. While, the proportions new born vaccinated $\psi$, natural death rate $\mu$, the proportion of individuals who do not get chance for screened at $H_{s}$ and will go to $L_{s}$ class $\varepsilon$, the Proportion of latently infected drug sensitive $\mathrm{TB}$ at early stage for treatment $p$, the recovery rates infectious individuals $\rho_{s}$ and $\rho_{r}$, the induced death rates $d_{s}$ and $d_{r}$; and of the portion of $E$ enter in to $I_{r}, v$ have negative impact on the transmission of TB disease.

From table 3 for the parameters of which the sensitivity index of $R_{e f f}(\mathrm{DS})$ and $R_{e f f}(\mathrm{MDR})$ has positive sign the effective reproduction number increase as those parameters increase and vise verse, while for those parameters of which sensitivity index of $R_{e f f}$ (DS) or $R_{\text {eff }}$ (MDR) has negative sign then the effective reproduction number increase as the parameters decrease and vise versa. The number of effective contact of susceptible or vaccinated individual with an infectious individual of both strains $\mathrm{c}$, the probability of transmission followed by the recovery rates infectious individuals are the most influential parameters in the spread and control of tuberculosis disease, this is because of that magnitude of the sensitivity indices $\Pi_{c}^{R_{e f f}}, \Pi_{\omega_{s}}^{R_{e f f}}, \Pi_{\rho_{s}}^{R_{e f f}}, \Pi_{\omega_{r}}^{R_{e f f}}$ are, $\Pi_{\rho_{r}}^{R_{e f f}}$ are maximum compared to others. The result of this study indicates that reducing the number of effective contact and increasing recovery rate have great role to control tuberculosis disease.

\section{Conclusion}

In this study we have presented and analyzed the two strain TB model with interventions: vaccination of newly born babies, screening of latently infected and treatments of infectious individuals for both strains of tuberculosis (drug sensitive and multi-drug resistance tuberculosis). We found that

$$
R_{e f f}(\mathrm{DS})=c \omega_{s}\left(\frac{\sigma \psi \mu+(\theta+(1-\psi) \mu)}{\theta+\mu}\right) \frac{(\alpha \varepsilon(1-p) \gamma \eta+\alpha(\gamma+\mu)(1-\varepsilon)(1-p))}{(\alpha+\mu)(\gamma+\mu)\left(\rho_{s}+\mu+d_{s}\right)} \text { and } R_{e f f}(\mathrm{MDR})=c \omega_{r}\left(\frac{\sigma \psi \mu+(\theta+(1-\psi) \mu)}{\theta+\mu}\right) \frac{(1-v) \delta}{(\delta+\mu)\left(\rho_{r}+\mu+d_{r}\right)}
$$

the effective reproduction numbers of drug sensitive and multi-drug resistance tuberculosis respectively. And, thus $R_{\text {eff }}=\max \left\{R_{\text {eff }}(\mathrm{DS}), R_{\text {eff }}(\mathrm{MDR})\right\} \quad$ is $\quad$ the effective reproduction number of the system (9)-(18). We have discussed on the existence of disease free equilibrium point, endemic equilibrium (drug-sensitive TB only endemic equilibrium, drug-resistance TB only endemic equilibrium and endemic equilibrium when both strains exist) points and presented the conditions that the local and global stability of those equilibrium points. We evaluated the numerical value of the reproduction numbers. Consequently, $R_{\text {eff }}(\mathrm{DS})=$ 1.03 and $R_{\text {eff }}(\mathrm{MDR})=4.78$, which show that the disease of both strain tuberculosis spread in the community and MDRTB spreads vastly in the society. The sensitivity analysis shows that the number of effective contact of susceptible or vaccinated individual with an infectious individual of both strains is the most influential parameter to change the reproduction number respectively.

\section{Recommendation}

In order to decrease the spread of both strains in the society, we recommend that the number of contact of susceptible individuals, c should be less than two. The 
second influential parameter to reduce the transmission of tuberculosis in Ethiopia is the recovery rate infectious individuals, $\rho_{r}$. In order to control the spreads of MDR-TB it needs to raise the value of $\rho_{r}$ over 2.8. The result of this study indicates that reducing the number of contact of susceptible or vaccinated individuals with an infectious and increasing recovery rate have great role to control tuberculosis disease.

\section{References}

[1] A. Jabbari and C. Castilo-Chaviz, A two-strain TB mode with multiple latent stages mathematical biosciences and engineering, 2016.

[2] B. K. Mishra, Mathematical model on pulmonary and multidrug-resistant tuberculosis patients with vaccination, Department of Applied Mathematics, Birla Institute of Technology, Mesra, Ranchi 8352015.

[3] C. P. Bhunu, A two strain tuberculosis transmission model with therapy and quarantine, 2010.

[4] D. Meressa Achieving high treatment success for multidrugresistant TB in Africa: initiation and scale-up of MDR TB care in Ethiopia - an observational cohort study, http://thorax.bmj.com/ on November 25, 2015.

[5] F. B. Agusto, Mathematical Model of MDR-TB and XDR-TB with Isolation and Lost to Follow-Up, 2015.

[6] A. Deribew, Tuberculosis Burden in Ethiopia from 1990 to 2016: Evidence from the Global Burden of Diseases, https://www.researchgate.net/publication/328602797, 2018.

[7] FDREM-H Ethiopian Public Health Institute, 2014, https://www.who.int/globalchange/resources/washtoolkit/review-of-policy-documents-on-climate-change-washand-public-health-in-ethiopia.pdf?ua $=1$.

[8] Federal Democratic Republic of Ethiopia Ministry of Health (EMH), 2018, https://www.medbox.org/national-strategicplan-tuberculosis-and-leprosy-control-2006-2013-ec-2013142020/download.pdf.

[9] Y. Yu, Dynamic model of tuberculosis considering multi-drug resistance and their applications, 2018.

[10] A. A. Yeketi, A Compartmental Model on the Effect of Quarantine on MDR-TB, International Journal of Mathematics and Computer Science, 2019.

[11] Implementation Guideline for GeneXpert MTB/RIF Assay in Ethiopia, 2014. https://www.medbox.org/et-guidelines-hivtb/implementation-guideline-for-gene-xpert-mtbrif-assay-inethiopia/preview.

[12] M. Al-arydah, Modelling the impact of treatment on tuberculosis transmission dynamics, International Journal of Biomathematics and Systems Biology, 2015.
[13] S. Eshetie, Tuberculosis treatment outcomes in Ethiopia from 2003 to 2016, and impact of HIV coinfection and prior drug exposure: A systematic review and meta-analysis, 2018.

[14] Z. B. Shimelis and T. T. Temesgen Dynamics of a Tuberculosis Model with Vaccination and Dual Treatments: a Mathematical model Analysis, e-ISSN: 2278-5728, p-ISSN: 2319-765X. Volume 15, Issue 4 Ser. II (Jul \{ Aug 2019), PP 47-60 www.iosrjournals.org, IOSR-JM.

[15] Z. Shuai, Global stability of infectious disease models using Lyapunov functions, society for industrial and applied mathematics, 2013.

[16] S. A. Gershgorin, Gershgorin's Circle Theorem. http://en.wikipedia.org, 1931.

[17] Federal Democratic Republic of Ethiopia Ministry of Health national, https://www.afro.who.int/publications/nationalstrategic-plan-tuberculosis-and-leprosy-control-2006-2013-ec201314-2020.

[18] Ethiopia Demographics Profile, 2019, https://www.indexmundi.com/ethiopia/demographics_profile. html.

[19] World Health Organization, Global tuberculosis report, https://www.who.int/tb/publications/global_report/gtbr2017_ main_text.pdf.

[20] B. Bewketu: Modeling Tuberculosis transmission dynamics in children and adults in the presence of vaccination (2011).

[21] M. Maliyani, P. M. Mwamtobe, S. D. Hove-Musekwa and J. M. Tchuenche: Modelling the role of diagnosis, Treatment and Health education on Multi-Drug resistant tuberculosis dynamics International Journal of Biomathematics and Systems Biology, 2015.

[22] Z. Gashu, The Yield of Community-Based "Retrospective" Tuberculosis Contact Investigation in a High Burden Setting in Ethiopia, DOI:10.1371/journal.pone.0160514, 2016.

[23] A. B. Gumel, Existence of multiple-stable equilibria for a multi-drug-resistant model of mycobacterium tuberculosis, mathematical biosciences and engineering, 2008.

[24] T. Girum, Epidemiology of multidrug-resistant tuberculosis (MDR-TB) in Ethiopia: a systematic review and meta-analysis of the prevalence, determinants and treatment outcome, Travel Medicine and Vaccines (2018).

[25] D. Okuonghae, and A. Korobeinikov, Dynamics of Tuberculosis: The Effect ofDirectObservation Therapy Strategy (DOTS) in Nigeria, MathematicalModeling of NaturalPhenomena, 2 (2007), 101-113.

[26] N. Chitnis, J. Hyman and J. Cusching, Determining important parameters in the spread of malaria through the sensitivity analysis of a mathematical model. Bulletin of Mathematical Biology 70 (2008) 12721296. 